\title{
CRISPR/Cas9-mediated gene knockin in the hydroid Hydractinia symbiolongicarpus
}

Steven M. Sanders ${ }^{1,2} \mathbb{B}$, Zhiwei Ma ${ }^{1,2}$, Julia M. Hughes ${ }^{1,2}$, Brooke M. Riscoe ${ }^{1,2}$, Gregory A. Gibson $^{3}$, Alan M. Watson ${ }^{3}$, Hakima Flici ${ }^{4}$, Uri Frank ${ }^{4}$, Christine E. Schnitzler ${ }^{5}$, Andreas D. Baxevanis ${ }^{6}$ and Matthew L. Nicotra ${ }^{1,2,7^{*}}$

\begin{abstract}
Background: Hydractinia symbiolongicarpus, a colonial cnidarian, is a tractable model system for many cnidarianspecific and general biological questions. Until recently, tests of gene function in Hydractinia have relied on laborious forward genetic approaches, randomly integrated transgenes, or transient knockdown of mRNAs.

Results: Here, we report the use of CRISPR/Cas9 genome editing to generate targeted genomic insertions in $H$. symbiolonigcarpus. We used CRISPR/Cas9 to promote homologous recombination of two fluorescent reporters, eGFP and tdTomato, into the Eukaryotic elongation factor 1 alpha (Eef1a) locus. We demonstrate that the transgenes are expressed ubiquitously and are stable over two generations of breeding. We further demonstrate that CRISPR/ Cas9 genome editing can be used to mark endogenous proteins with FLAG or Strepll-FLAG affinity tags to enable in vivo and ex vivo protein studies.

Conclusions: This is the first account of CRISPR/Cas9 mediated knockins in Hydractinia and the first example of the germline transmission of a CRISPR/Cas9 inserted transgene in a cnidarian. The ability to precisely insert exogenous DNA into the Hydractinia genome will enable sophisticated genetic studies and further development of functional genomics tools in this understudied cnidarian model.
\end{abstract}

Keywords: Genome editing, Immunohistochemistry, Cnidaria, Invertebrate, Model organism, Transgenic, FLAG, eGFP, tdTomato, P2A

\section{Background}

Cnidarians are a phylum of morphologically simple, primarily marine invertebrates. They have long served as experimental models in the fields of developmental biology, symbiosis, and self/non-self recognition (reviewed in [1-4]). More recently, cnidarians have generated interest as models for regenerative and stem cell biology [5-7], neurobiology $[8,9]$, biophysics $[10,11]$ and aging $[12,13]$. As the closest outgroup to bilaterians $[14,15]$, cnidarians also provide context for our understanding of how fundamental biological processes have evolved in other animals, including humans. Indeed, cnidarian genomes are remarkably similar to the human genome in terms of gene content and structure [16, 17]. Cnidarian

\footnotetext{
*Correspondence: matthew.nicotra@pitt.edu

'Department of Surgery, Thomas E. Starzl Transplantation Institute, University of Pittsburgh, Pittsburgh, PA, USA

${ }^{2}$ Pittsburgh Center for Evolutionary Biology and Medicine, University of Pittsburgh, Pittsburgh, PA, USA

Full list of author information is available at the end of the article
}

genomes also possess many human disease genes not found in other animal models [18]. For these reasons, there is an increasing demand for tools allowing direct tests of gene function in cnidarian species.

Hydractinia is a genus of colonial marine hydroids and a rapidly maturing model system for cnidarian genetics and functional genomics. Like the two most commonly used cnidarian models, Hydra and Nematostella, Hydractinia has played a key role in our understanding of the molecular basis of development and tissue regeneration [19-22]. Because Hydractinia diverged from Hydra 306428 million years ago and Nematostella 570-635 million years ago [23-26] (divergence times roughly equivalent to the human/frog and human/hagfish splits, respectively [27]), genetic studies that include these three taxa increase the phylogenetic resolution with which we can understand the evolution of gene function. In addition, Hydractinia exhibits traits that are difficult or impossible to study at the molecular level in other cnidarians, including a pluripotent stem cell lineage called interstitial cells (i-cells) [6], 
coloniality [28], morphological polymorphism [20, 29], and allorecognition [4].

From a practical standpoint, Hydractinia offers several advantages. It is small in size and inexpensive to culture. Robust laboratory strains for one Hydractinia species $(H$. symbiolongicarpus) are available (Nicotra ML, personal communication), and a project to sequence its genome is underway [30]. It is transparent, facilitating in vivo microscopic observations throughout the animal at all developmental stages. It is also simple to breed because it spawns daily in response to a light cue, generates embryos that are easy to manipulate, grows into adults that are sessile and dioecious, and has a three-month generation time (comparable to mice and zebrafish). In addition, colonies can be clonally replicated and do not appear to age in the lab, allowing researchers to work with the same animal for years. These features are one reason that $H$. symbiolongicarpus is the only cnidarian in which a forward genetic approach has been used to identify the genetic basis of a phenotype [31, 32]. Reverse genetic techniques are also possible in Hydractinia, including RNA interference (RNAi) [21, 33, 34], morpholinos [35], and the creation of transgenics via the random integration of exogenous DNA into the genome [22, 34, 36].

One bottleneck in performing functional genomics in Hydractinia and other cnidarians has been the inability to create heritable genetic modifications leading to true gene knockouts or knockins. With the advent of CRISPR/Cas9 genome editing, this goal is now within reach. To date, CRISPR/Cas9 has been used to disrupt genes in several cnidarians, including Nematostella [37-40], Clytia [41], Acropora [42], and Hydractinia [43]. These studies have each employed embryonic injection of one or more guide RNAs (sgRNAs) to disrupt a gene of interest and then evaluated phenotypes during the development of these injected embryos or the adult animals derived from injected embryos. In addition, there has been one study in which a fluorescent reporter was knocked into a gene in Nematostella [40]. Germline transmission of a CRISPR/ Cas9 mediated genome edit has yet to be achieved in any cnidarian.

Here, we report the first use of CRISPR/Cas9 genome editing to generate knockins in H. symbiolongicarpus. Specifically, we use CRISPR/Cas9 to knock fluorescent reporters and small affinity tags into the endogenous Eukaryotic elongation factor 1 alpha (Eefla) locus. The transgenes were successfully transmitted through the germline to create fully transgenic animals and were stable over two generations of breeding. In addition, we demonstrate the utility of tagging the endogenous protein with two different affinity tags to perform in vivo and ex vivo protein studies. The ability to generate targeted gene knockins greatly increases the feasibility of studying the functions of cnidarian genes in their endogenous genomic environment, which can include complex cis and trans regulatory elements. It will also enable researchers to more efficiently create knockouts with fluorescent gene traps, to test hypotheses of protein function through targeted point mutations, and to engineer conditional transgenics that will allow gene expression to be controlled in site-, cell-, and/or time-specific ways.

\section{Results \\ Identification of Hydractinia Eef1a and parental genotyping}

Published and unpublished work from our laboratories has shown it is possible to create point mutations, small indels, and large deletions in the Hydractinia genome by injecting Cas9:sgRNA protein:RNA complexes into zygotes $[43,44]$. In the present study, we sought to test whether we could use CRISPR/Cas9 mediated homologous recombination to introduce exogenous DNA encoding fluorescent proteins into the Hydractinia genome. To test this, we focused on editing the Eefla locus because it is a highly expressed housekeeping gene and would enhance our chances of visualizing the insertions.

To identify the Hydractinia Eefla locus, we used previously published $H$. symbiolongicarpus RNA-Seq data [45] in conjunction with a draft genome assembly for a male colony, 291-10 [30]. Because Hydractinia are diploid and 291-10 was bred from two animals from an outcrossing population, we expected it to carry two distinct Eef1a alleles, which we searched for and found in the mRNA and genome sequences. Each allele encodes the same 465 amino acid peptide, spans $\sim 2.5 \mathrm{~kb}$ of genomic sequence, and is comprised of four exons (Fig. 1a) (GenBank accession numbers MH513671 and MH513672). The top hits resulting from BLASTP searches of these Eef1a peptides against the NCBI refseq_protein database were to Eefla proteins from Trichoplax adhaerens (87\% identity), Branchiostoma floridae (85\% identity), and Crassostrea virginica (84\% identity).

Our microinjection experiments would ultimately require embryos, which we planned to generate by crossing 291-10 to a female colony, 295-8, which is a half-sibling of 291-10 (Additional file 1: Figure S1). Because this female was likely to be heterozygous at Eefla, we also genotyped it at the targeted region of Eef1a. We PCR-amplified the genomic region encoding exons 3-4 of Eefla, which yielded a $2.0 \mathrm{~kb}$ and a $2.4 \mathrm{~kb}$ amplicon. Each amplicon was cloned and sequenced, revealing a $\sim 400 \mathrm{bp}$ indel downstream of the Eef1a 3'UTR (Additional file 2). We concluded these sequences represented the two maternal Eef1a alleles. Aligning the four Eef1a alleles (two alleles from each parent of the planned cross) revealed several sequence variants but, 


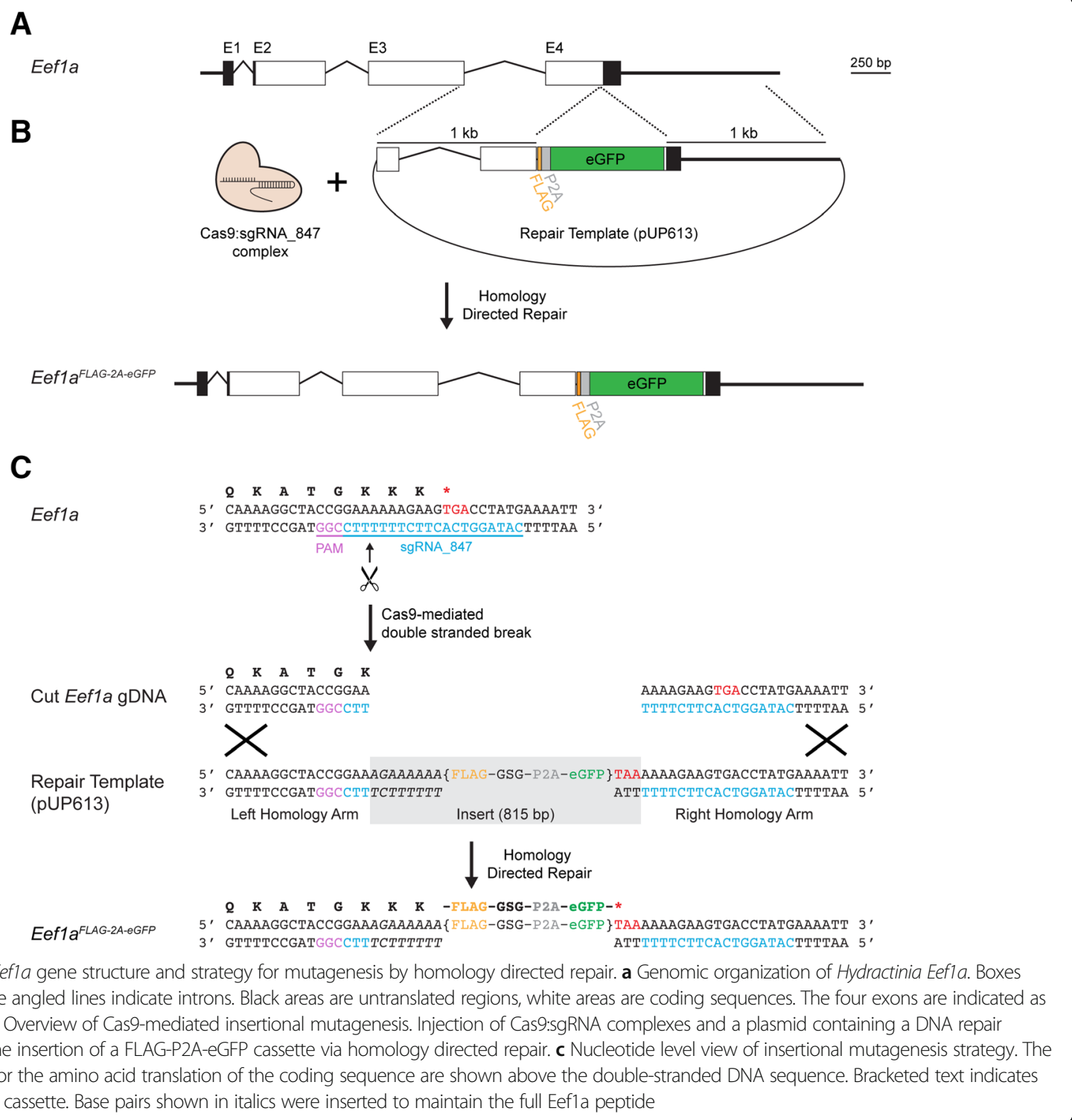

as expected for a highly conserved housekeeping gene, identical coding sequences (Additional file 3).

\section{CRISPR/Cas9-mediated homologous recombination at the Eef1a locus}

Our first goal was to test whether we could insert a transgene encoding an affinity tag and fluorescent reporter into the 3' end of Eef1a via Cas9-mediated homology directed repair (Fig. 1b). To do this, we identified a NGG PAM site 14 bp upstream of the stop codon and used it to design a 20 bp sgRNA (sgRNA_847, Fig. 1c) that was conserved across the four Eef1a alleles (Additional file 3). Local BLASTs of this sgRNA_847 sequence against the draft genome revealed no obvious off-target matches outside of Eefla in the genome. We then created a plasmid to act as the repair template for homology-directed repair. This plasmid, pUP613 (Additional file 4), consisted of two $1000 \mathrm{bp}$ homology arms matching the sequences immediately before and after the predicted Cas 9 cut site of one Eef1a allele from colony 291-10. Between these arms, we inserted $8 \mathrm{bp}$ to complete the Ef1a coding sequence (italicized in Fig. 1c). Silent mutations in these 8 bp ensured the plasmid would not be targeted by Cas9:sgRNA_847 complexes. This was followed by sequences encoding a FLAG peptide, a Gly-Ser-Gly linker peptide, a P2A self-cleaving peptide, eGFP, and a stop codon, all codon-optimized for Hydractinia (Fig. 1c). When correctly inserted into the 3' end of Eef1a, we predicted this construct would create a mutant allele, Eef1aFLAG-P2A-eGFP, resulting in the expression of a Eefla-FLAG protein and a separate eGFP molecule that would act as a visual marker. Because the repair template did not include 
a promoter or splice acceptor site, untargeted integration of the plasmid into the genome would be unlikely to result in eGFP expression.

To test our construct, we injected zygotes with an injection cocktail containing $200 \mathrm{ng} / \mu \mathrm{l}$ repair template pUP613 and $1 \mu \mathrm{g} / \mu \mathrm{l}$ Cas9 complexed with $500 \mathrm{ng} / \mu \mathrm{l}$ sgRNA_847 (Fig. 2a). Pilot experiments comparing injected and non-injected embryos revealed an impact of injection on viability (Additional file 1: Figure S2A). Despite this effect, we recovered 321 viable injected embryos (from three independent experiments), of which 14 (4.4\%) were $\mathrm{eGFP}^{+}$(Additional file 1: Figure S2B). The location of $\mathrm{eGFP}^{+}$expression ranged from several endodermal cells to long streaks of $\mathrm{eGFP}^{+}$cells and remained present up to $144 \mathrm{~h}$ post fertilization (hpf) (Fig. 2b). In control experiments in which we injected the repair template alone, we did not observe any eGFP ${ }^{+}$cells (100 viable, injected embryos). A parallel set of experiments conducted with the same strains of $H$. symbiolongicarpus and the same injection cocktail in the Frank laboratory at the National University of Ireland, Galway also generated $\mathrm{eGFP}^{+}$colonies (unpublished observations).

We subsequently injected embryos and settled only those larvae with many eGFP ${ }^{+}$cells $(n=36)$. In all cases, we found that $\mathrm{eGFP}^{+}$larvae metamorphosed into primary polyps with eGFP ${ }^{+}$cells. As they developed into juvenile colonies, several of these animals lost eGFP expression. These were typically primary polyps that possessed few $\mathrm{eGFP}^{+}$cells after metamorphosis. In 15 colonies, however, eGFP expression was stably maintained, albeit mosaic in most colonies.

In one $\mathrm{eGFP}^{+}$colony, 347-10, we observed nearly ubiquitous eGFP expression at sexual maturity. In particular, we noted high levels of eGFP fluorescence in all developing oocytes (Fig. 2c), raising the possibility that the Eef1a ${ }^{\text {FLAG-P2A-eGFP }}$ allele might be present in the germline. To test this, we backcrossed colony 347-10 to its male parent (Fig. 2a). At $24 \mathrm{hpf}, 100 \%$ of larvae obtained from this cross were $\mathrm{eGFP}^{+}$(Fig. 2d), although some appeared slightly dimmer than others (Additional file 1: Figure S3A). By 72 hpf, we could clearly distinguish $\mathrm{eGFP}^{+}$from eGFP dim larvae (Fig. 2e; Additional file 1: Figure S3B), suggesting that the $\mathrm{eGFP}^{+}$had received a germline transmitted Eef1a $a^{F L A G-P 2 A-e G F P}$ allele, while GFP $^{\text {dim }}$ fluoresced due to maternal loading of either eGFP mRNA or protein in the oocyte. This low level of eGFP signal remained in the larvae up to 7 days post-fertilization, while eGFP+ larvae accumulated bright eGFP signal, particularly in the endodermal cells (Fig. 2f; Additional file 1: Figure S3C). The eGFP ${ }^{+}$larvae that we settled developed into $\mathrm{eGFP}^{+}$adults with high levels of eGFP expression, while eGFP ${ }^{\text {dim }}$ larvae developed into colonies without eGFP expression.
To further assess the stability of this transgene, we grew several of these $\mathrm{eGFP}^{+}$colonies to sexual maturity and never observed loss of eGFP expression. We then selected a male and a female (Fig. 2g and h, respectively), backcrossed them to their parents, and crossed them to each other (Fig. 2a). At $24 \mathrm{hpf}$, larvae resulting from the backcross of the male $\mathrm{eGFP}^{+}$colony could readily be classified as $\mathrm{eGFP}^{+}$or $\mathrm{eGFP}^{-}$(not shown). In contrast, larvae resulting from both crosses involving the female $\mathrm{eGFP}^{+}$colony displayed either high or low eGFP signal similar to that observed coming from the founder colony, 347-10. This phenotype resolved into $\mathrm{eGFP}^{+}$and eGFP $^{\text {dim }}$ classes by 72 hpf. At 96 hpf, we counted the number of $\mathrm{eGFP}^{+}$and $\mathrm{eGFP}^{\mathrm{dim}}$ or $\mathrm{eGFP}^{-}$larvae in each cross and confirmed that the inheritance pattern of eGFP expression was consistent with mendelian inheritance of a single Eef $1 a^{F L A G-P 2 A-e G F P}$ allele from colonies 354-3 and 354-5 (Table 1).

To test for potentially deleterious fitness effects of the Eef1 $a^{\text {FLAG-P2A-eGFP }}$ allele, we settled $44 \mathrm{eGFP}^{+}$and 50 $\mathrm{eGFP}^{-}$larvae from the 357 cross and followed them for 4 weeks. We observed no difference in survival or total area of the mat (a proxy for colony size) between the two groups (Fig. 2i-j). To date, we have continued to culture five animals from the 357 population for 7 months and observed stable eGFP expression in each one.

To determine whether we had correctly inserted the FLAG-P2A-eGFP cassette into the Eefla locus, we designed PCR primers to amplify the transgene from $\mathrm{eGFP}^{+}$animals. Repeated attempts to amplify the expected 3232 bp cassette from eGFP $^{+}$colonies with primers Pr845 and Pr846 were unsuccessful. We concluded this was likely due to preferential amplification of the shorter, unedited Eef1a allele. We therefore developed a strategy to amplify each side of the inserted transgene. To avoid amplifying repair template DNA that may have integrated elsewhere in the genome, we designed each primer pair such that one primer annealed to the endogenous Eef1a locus outside the region matching the homology arms in pUP613 and the other to the insert (Fig. 3a and Additional file 1: Table S1). Using primers flanking the $5^{\prime}$ homology arm, we were able to amplify a band of the expected size from the founder (347-10) and its $\mathrm{eGFP}^{+}$progeny, but not their $\mathrm{eGFP}^{-}$siblings (Fig. 3b). We cloned and sequenced these amplicons and found that they matched the expected sequence of the Eef1a $a^{F L A G-P 2 A-e G F P}$ allele (Additional file 5). Using primers flanking the 3' homology arm, we were also able to amplify products from $\mathrm{eGFP}^{+}$ colonies, but these were smaller than expected. Cloning and sequencing them revealed that they matched expected sequence of the Eef1a $a^{F L A G-P 2 A-e G F P}$ allele except for a 681 bp deletion downstream of the Eef1a 3' UTR. These experiments confirmed that colonies $354-3$ and 354-5 


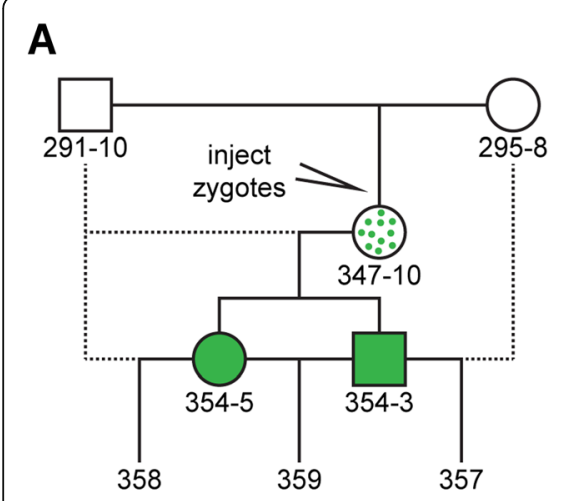

\section{B}
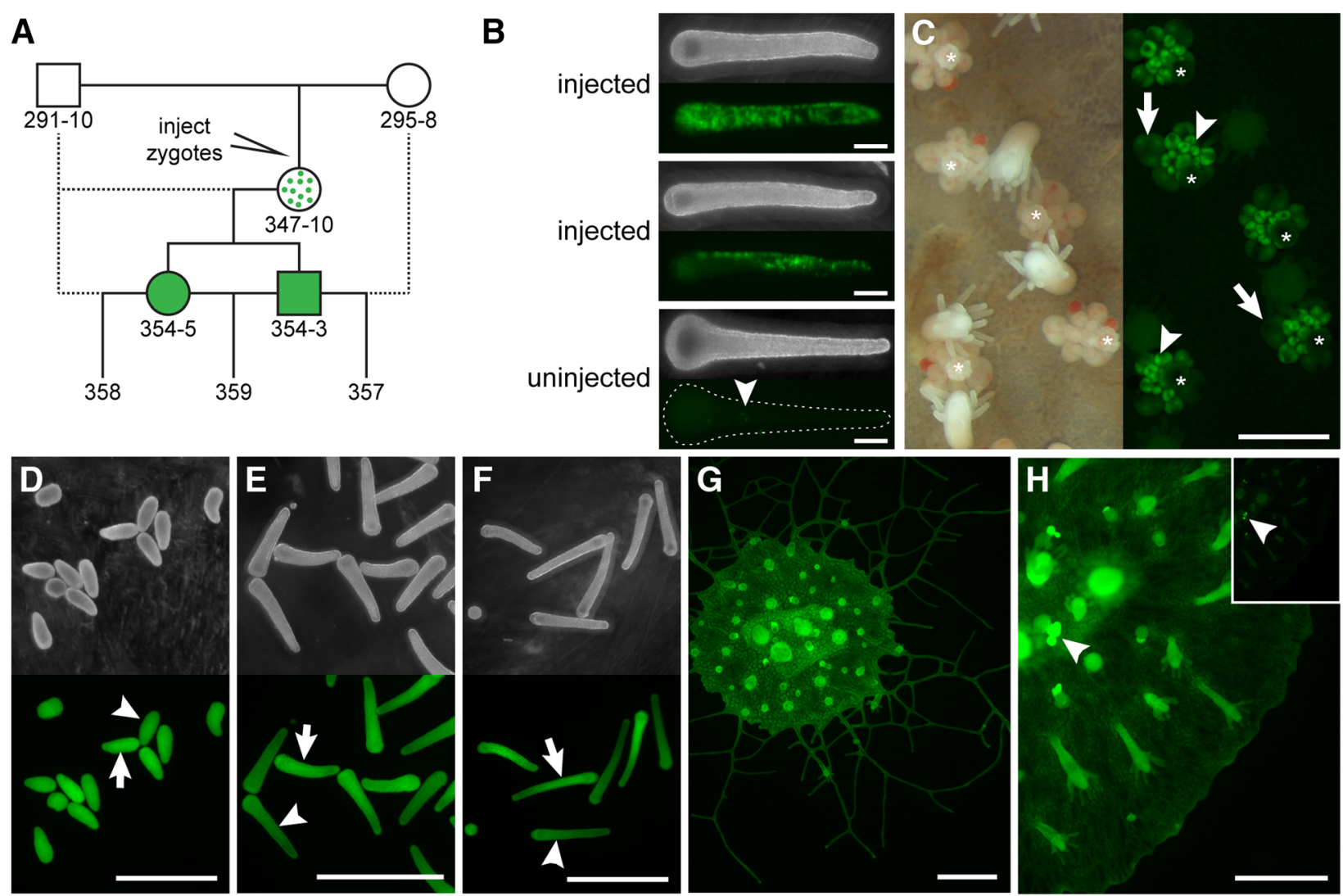

I

colony survival

$\mathbf{J}$
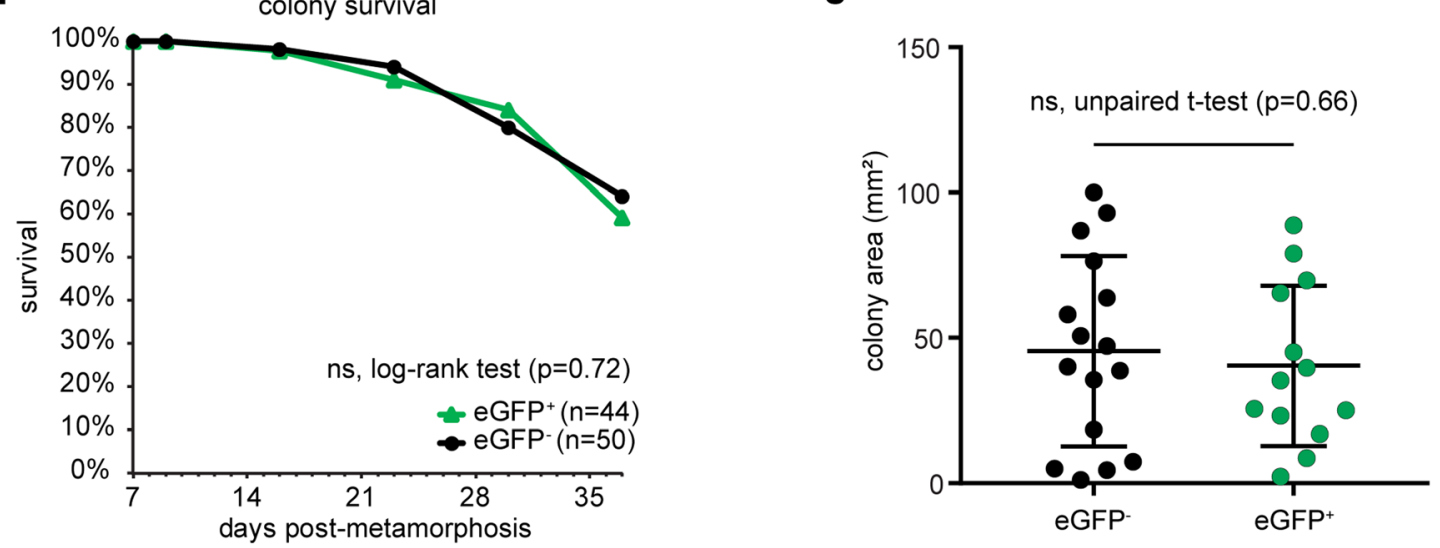

Fig. 2 eGFP expression following Cas9 injection. a Breeding strategy and pedigree of eGFP ${ }^{+}$colonies described in this paper. Colony $347-10$ is a founder derived from an injected embryo and is assumed to be a mosaic of edited eGFP' cells and wild-type eGFP ${ }^{-}$cells. Dotted lines indicate backcrosses. $\mathbf{b}$ Larvae injected with Cas9:sgRNA_847 complexes + repair template pUP613 at 144 hpf post-injection, showing mosaic expression of eGFP. An uninjected 144 hpf larvae is shown for comparison. Specks of green auto-fluorescence are common in uninjected larvae (arrowhead) but are easily distinguished from eGFP expression. c Left panel: dark-field image of colony 347-10, showing gonozooids (white asterisk). Right panel: Fluorescence micrograph of the same area of colony 347-10 showing eGFP expression. Developing oocytes (arrowheads) are intensely fluorescent, but become dim as they mature (arrows). $\mathbf{d}$ Darkfield (top) and fluorescence (bottom) micrograph of $24 \mathrm{hpf}$ embryos from the backcross of 347-10 to 291-10, beginning to show slightly dimmer eGFP signal in some larvae (arrowhead) compared to others (arrow). e 72 hpf embryos from the same cross. eGFP (arrow) and eGFPdim (arrowhead) larvae are now evident. $\mathbf{f} 168$ h-old embryos. The distinction between eGFP (arrow) and eGFPdim (arrowhead) larvae is even more substantial. $\mathbf{g}$ Fluorescence micrograph of an explant of colony 354-3. h Fluorescence micrograph of an explant of colony 354-5. Image input and output levels were adjusted to reveal eGFP fluorescence in stolonal mat. Inset: Image with levels scaled automatically in NIS-elements, showing intense fluorescence of developing oocytes (arrowhead). i Survivorship of eGFP ${ }^{+}$and GFP $^{-}$colonies from population 357. $\mathbf{j}$ Mat area measurements of colonies from population 357. All scale bars $=1 \mathrm{~mm}$ 
Table 1 Mendelian inheritance of eGFP expression in testcrosses

\begin{tabular}{|c|c|c|c|c|c|c|}
\hline \multirow[t]{2}{*}{$\operatorname{Cross}^{\mathrm{a}}$} & \multirow[t]{2}{*}{ Parents } & \multicolumn{2}{|l|}{ Hypothesized Genotypes } & \multirow[b]{2}{*}{$N^{c}$} & \multirow{2}{*}{$\begin{array}{l}\text { Phenotype }^{\mathrm{d}} \\
\mathrm{eGFP}^{+} / \mathrm{eGFP}\end{array}$} & \multirow[b]{2}{*}{ Goodness of fit } \\
\hline & & Parental (male $\times$ female) & Offspring $^{\mathrm{b}}$ & & & \\
\hline 357 & $\begin{array}{l}354-3 \\
\times \\
295-8\end{array}$ & $\begin{array}{l}\text { Eefla } \\
x \\
\text { EeflaG-P2A-eGFP/+ }^{+/+}\end{array}$ & $\begin{array}{l}\text { Eef1a } a^{\text {FLAG-P2A-EGFP/+ }} \\
\text { Eefla }^{+/+}(1: 1)\end{array}$ & 263 & $136 / 127$ & $x^{2}=0.647, p=0.58$ \\
\hline 358 & $\begin{array}{l}291-10 \\
\times \\
354-5\end{array}$ & $\begin{array}{l}\text { Eef1 }^{+/+} \\
\times \\
\text {Eef1a }^{\text {FLAG-P2A-GGFP/+ }}\end{array}$ & $\begin{array}{l}\text { Eef1 } a^{\text {FLAG-P2A-eGFP/+ }} \\
\text { Eefla }^{+/+}(1: 1)\end{array}$ & 124 & $71 / 53$ & $x^{2}=0.018, p=0.11$ \\
\hline 359 & $\begin{array}{l}354-3 \\
\times \\
354-5\end{array}$ & $\begin{array}{l}\text { Eef1a } a^{\text {FLAG-P2A-EGFP/+ }} \\
\times \\
\text { Eef1a }^{\text {FLAG-P2A-EGPP/+ }}\end{array}$ & 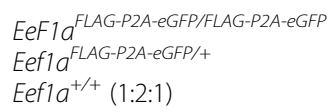 & 111 & $81 / 30$ & $x^{2}=0.777, p=0.62$ \\
\hline
\end{tabular}

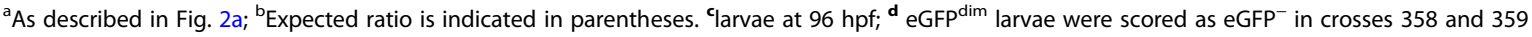

inherited the same Eef1a $a^{F L A G-P 2 A-e G F P}$ allele from the founder colony, 347-10, but do not entirely rule out the possibility that $347-10$ was a mosaic possessing multiple edited Eef1a alleles. Taken together, these results show that CRISPR/Cas9-mediated homologous recombination can be used to introduce exogenous DNA into target sites in the Hydractinia genome, and that these sequences can be stably inherited over multiple generations.

\section{The FLAG octopeptide enables specific tagging of endogenous proteins in Hydractinia}

One potential use for CRISPR/Cas9 genome editing is to tag endogenous proteins of interest with short epitopes to facilitate in vivo and in vitro protein detection via immunolocalization and western blotting. This strategy could obviate the laborious task of creating and validating antibodies to target proteins for such studies. An additional advantage of using small epitope tags instead of fluorescent fusion proteins is that short tags are less likely to interfere with protein stability and function. To test whether we can specifically detect FLAG-tagged proteins in Hydractinia using commercially available reagents, we isolated total protein from transgenic and nontransgenic colonies and probed them with an anti-FLAG monoclonal antibody (M2) in western blots (Fig. 4a). We observed a strong, specific signal in colonies expressing the Eef1a-FLAG protein, with no detectable background in colonies expressing only untagged Eefla. The absence of additional bands at the expected size of the intact Eef1a-FLAG-P2A-eGFP protein $(80.8 \mathrm{kDa})$, even after $60 \mathrm{~min}$ of exposure to film, also demonstrated that the P2A self-cleaving peptide can efficiently separate two peptides in vivo in Hydractinia.

To test whether the FLAG octopeptide would be suitable for immunolocalizations, we probed whole mounts of transgenic and non-transgenic gastrozooids with the same anti-FLAG antibodies. We observed strong but diffuse staining in the transgenic polyps (Fig. 5a-c and Additional files 6 and 7), which was consistent with ubiquitous cytoplasmic expression of Eefla-FLAG. In addition, we noted cells with large nuclei and a polygonal morphology sparsely distributed within the body column (Fig. 5c-d). The

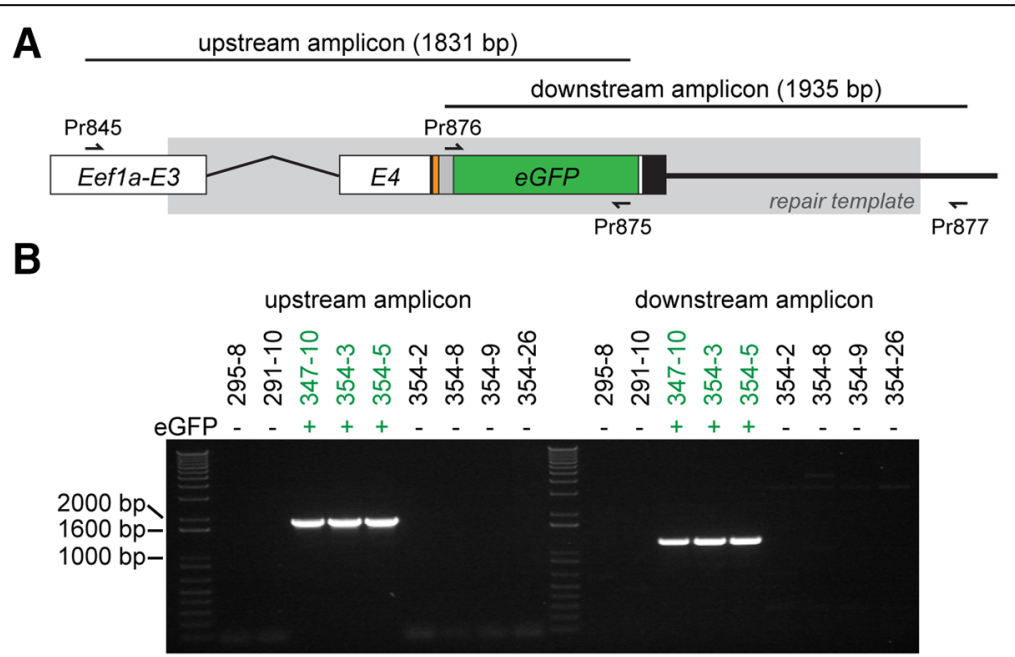

Fig. 3 Validation of homologous recombination at the Eef1a locus. a Schematic of the expected sequence for the edited region of Eef1 $a^{F L A G-P 2 A-e G F P}$. Exons are abbreviated E3 and E4. Sequence matching the repair template is shown in gray box. PCR primers are shown as small arrows. Coding sequence is colored as in Fig. 1. b Amplification of upstream and downstream regions of Eef1a ${ }^{\text {FLAG-P2A-EGFP }}$ from eGFP ${ }^{+}$and eGFP ${ }^{-}$Colonies $^{-}$ 


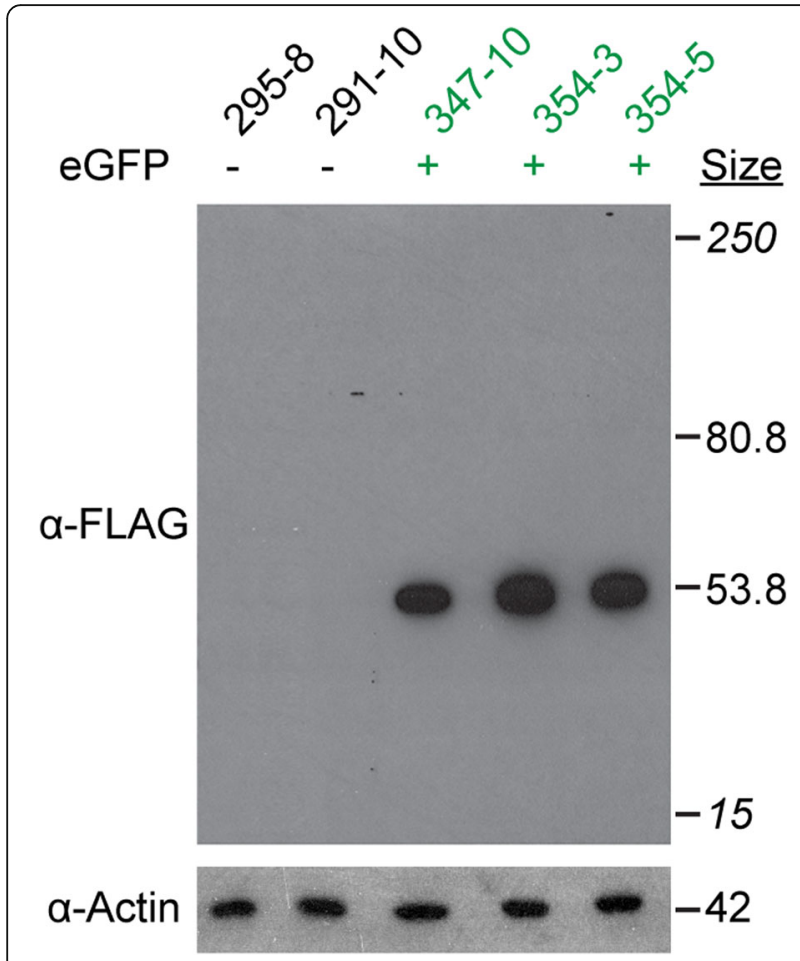

Fig. 4 Specificity of the FLAG octopeptide in Hydractinia. Western blot of total protein lysates from Eef1a ${ }^{\text {FAGP2AeGFP }}$ transgenic and nontransgenic colonies. Sizes in italics are the top and bottom bands of the molecular size marker

morphology of these cells was similar to that reported for Hydractinia interstitial stem cells (i-cells) (e.g., [22]). In contrast, we observed very little signal from the non-transgenic control (Fig. 5e-g and Additional files 8 and 9). This signal was dramatically reduced in secondary-only controls (Additional file 1: Figure S4), indicating it was likely the result of minimal non-specific staining from the FLAG antibody. These results suggest that tagging endogenous proteins with FLAG epitope tags could be a viable strategy for immunolocalization, provided appropriate controls are performed.

\section{Germline transmitted Eef1 $a^{F L A G-P 2 A-E G F P}$ is expressed without evidence of variegated expression}

Because Eefla is a constitutively expressed housekeeping gene, we expected to observe eGFP expression in all cells at all developmental stages. However, previous work has shown that germline transmitted, randomly integrated transgenes driven by promoters of housekeeping genes can display an expression pattern in which cells appear to either stochastically express or not express the transgene [36]. The mechanism responsible for this variegated expression pattern is unknown, but for transgenes driven by actin promoters, it can persist even after two generations of germline transmission (ML Nicotra, unpublished data).
To determine whether the germline transmitted Eef1 $a^{F L A G-P 2 A-e G F P}$ allele would exhibit similar variegated expression, we investigated its expression in detail. Multiphoton imaging of intact gastrozooids, gonozooids, stolons, and mat edges revealed no evidence of variegated expression in either the epidermal or gastrodermal cell layers (Fig. 6a-g, j, k and Additional files 10,11,12,13,14 and 15). The intensity of eGFP signal did not allow us to determine whether expression was uniform across all cell types. We also investigated $120 \mathrm{hpf} \mathrm{eGFP}^{+}$larvae from the 357 cross and found robust eGFP expression in all ectodermal and endodermal cells (Fig. 6h-i and Additional files 16 and 17). We therefore concluded that, as expected, the Eef1 $a^{\text {FLAG-P2A-eGFP }}$ transgene is at least expressed in all epithelial cells, with no detectable evidence of variegated expression.

\section{In vivo expression and function of alternative affinity tags and fluorescent reporters}

While the FLAG epitope was effective for immunoblotting and immunolocalization studies, repeated attempts to immunoprecipitate the tagged Eefla protein failed. Therefore, we decided to test the StrepII-FLAG tandem affinity purification (SF-TAP) tag (Fig. 7a) [46]. The SF-TAP tag is composed of a doublet Strep II tag followed by a single FLAG peptide and was chosen because it would potentially enable efficient purification of tagged proteins and their interaction partners via tandem affinity purification [46]. In addition, we replaced the eGFP with tdTomato because it is one of the brightest fluorescent proteins available [47] and can be used in combination with eGFP during imaging. The resulting repair template (pUP660; Additional file 18) was expected to create an Eef1a-SF peptide and a separate tdTomato peptide.

We co-injected pUP660 with Cas9:sgRNA_847 complexes into zygotes from parents $291-10$ and 295-8 (Fig. 7b). Similar to previous experiments, we observed tdTomato ${ }^{+}$cells in a portion of the injected embryos at $72 \mathrm{hpf}$ and selected only those larvae with high levels of tdTomato expression for metamorphosis into colonies. Of 38 such colonies, one colony, 350-10, developed tdTomato ${ }^{+}$eggs. In contrast to colony 347-10, however, not all eggs were fluorescent, even within the same gonophore (Fig. 7c-d). We backcrossed this colony to 291-10 and obtained several tdTomato ${ }^{+}$colonies.

To validate correct insertion of the SF-P2A-tdTomato cassette, we attempted to amplify regions upstream and downstream of the cassette using a similar PCR-based strategy to that employed for Eef1a ${ }^{\text {FLAG-P2A-eGFP }}$ (Fig. 7e). For the upstream region, we obtained bands of the expected size from tdTomato ${ }^{+}$colonies (Fig. $7 f$ ), which we cloned and sequenced and found identical to the expected sequence of the edited Eef1 $a^{S F-P 2 A-t d T o m}$ allele (Additional file 19). In contrast, we were unable to amplify the 

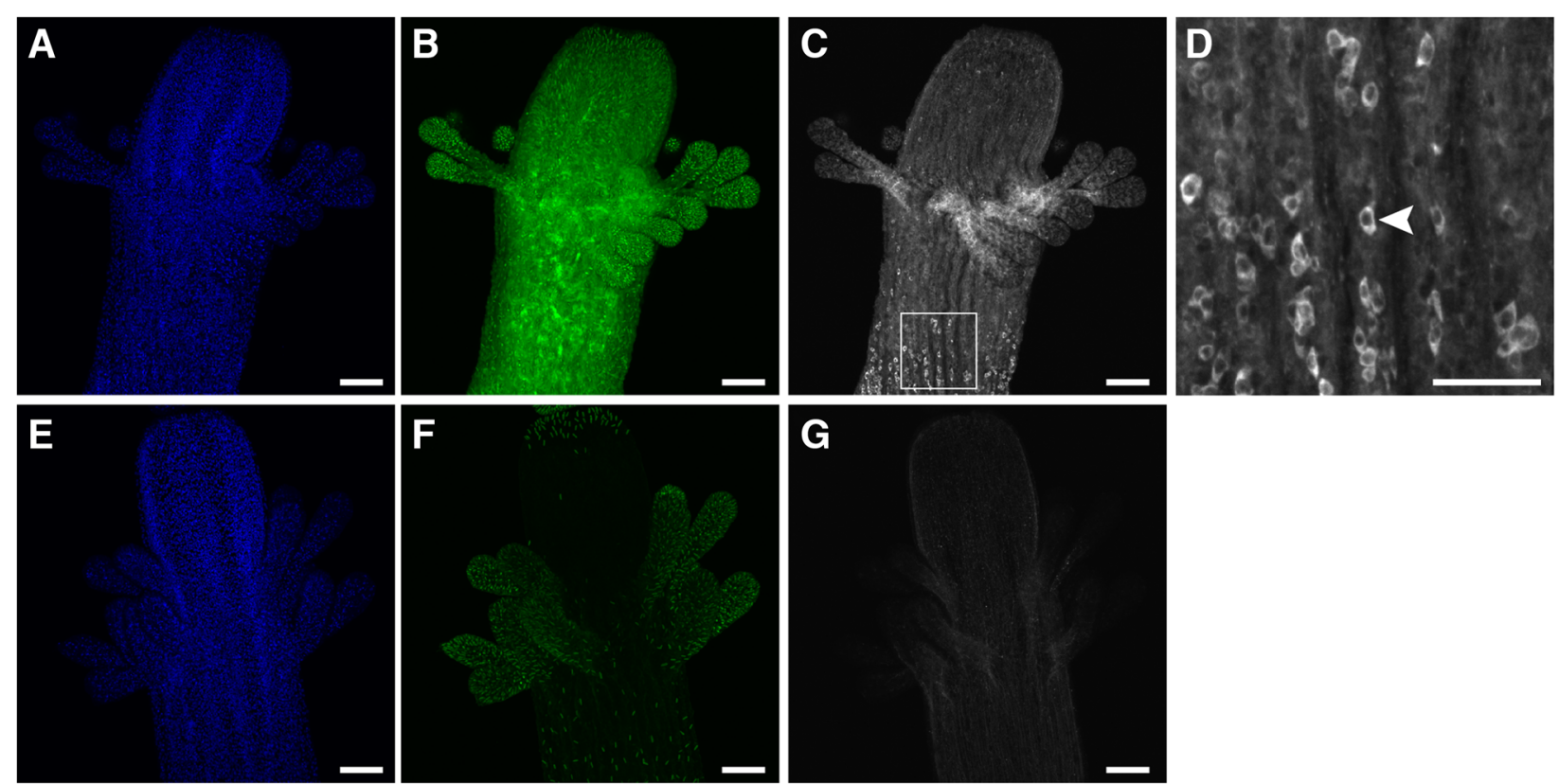

Fig. 5 Immunolocalization of Eef1a-FLAG. a-d Eef1 a ${ }^{F L A G-P 2 A-E G F P}$ transgenic gastrozooids fixed, permeabilized, and stained with anti-FLAG primary antibodies and Alexa 647 conjugated secondary antibodies, followed by DAPI, and imaged on a laser scanning confocal microscope. (a, b) DAPI staining and eGFP expression in the transgenic gastrozooid, respectively. c Anti-FLAG staining in the transgenic gastrozooid, showing strong, diffuse signal throughout with sparse, intensely stained cells. d An inset of panel C showing intensely stained cells with polygon-like morphology (e.g., arrowhead). e-g Nontransgenic controls imaged and analyzed with identical settings. (e, f) DAPI staining and green fluorescence, respectively. Nematocyst capsules exhibit bright autofluorescence. $\mathbf{g}$ Anti-FLAG staining, showing faint background staining. Scale bars $=100 \mu \mathrm{m}$, except for (d), which is $50 \mu \mathrm{m}$

downstream region, despite testing 11 PCR primers in 14 pairwise combinations (Fig. $7 \mathrm{~g}$ ). These results led us to conclude we had successfully created the Eef1a 1 SF-P2A-tdTom mutant allele and, since tdTomato was functional, that the sequence was intact at least through the stop codon. We could not, however, confirm the sequence of the downstream genomic region.

In culturing colonies from the 355 population, we also observed a marked fitness defect associated with the Eef1a ${ }^{S F-P 2 A-t d T o m}$ allele. Whereas tdTomato ${ }^{-}$larvae metamorphosed and grew into robust colonies, their tdTomato $^{+}$siblings required meticulous hand-feeding for several weeks, died at a high rate, and grew slowly. Despite this defect, we were able to obtain enough tissue to test the suitability of the SF-TAP tag for in vivo protein studies.

We first tested whether we could detect both tags via in western blots on total cell lysates of tdTomato ${ }^{+}$and tdTomato $^{-}$colonies. Using anti-FLAG antibodies, we observed a band of the expected molecular weight for an Eef1a protein bearing a C-terminal SF-TAP tag in an animal bearing a germline transmitted allele, but no signal in the mosaic founder (Fig. 8a). Using an anti-StrepII monoclonal antibody, we similarly observed a strong signal from the fully transgenic colony and no signal from the mosaic founder. (Fig. 8b). After over-exposure on film, however, a faint band appeared for the founder, likely reflecting the small number of properly edited cells in this colony (Fig. 8b). In addition, we observed a $15 \mathrm{kDa}$ band in all colonies, suggesting this antibody cross-reacts with some variants of an endogenous Hydractinia protein. These results further confirmed we had, indeed, created the Eef1a $a^{S F-P 2 A-t d T o m}$ allele, despite our inability to amplify the downstream genomic region via PCR.

Finally, to test whether we could use the SF-TAP tag to purify proteins from the Hydractinia proteome, we harvested tissue from transgenic and non-transgenic colonies, prepared whole cell lysates, incubated them with Strep-Tactin conjugated agarose beads, and eluted the bound protein. SDS-PAGE of the elutions followed by coomassie staining revealed a band of the expected size for Eef1a-SF from transgenic but not control colonies (Fig. 8c). We also observed a faint band smaller than the expected band, which may be a protein that specifically or non-specifically binds to Eef1a (Fig. 8c). To test the efficiency of the pull-down, we also probed whole cell lysates before and after the pull down with anti-FLAG antibodies and observed a complete loss of FLAG signal, indicating complete capture of the Eef1a-SF protein (Fig. 8d). These results demonstrate the suitability of the StrepII tag to purify in vivo tagged proteins from Hydractinia. 


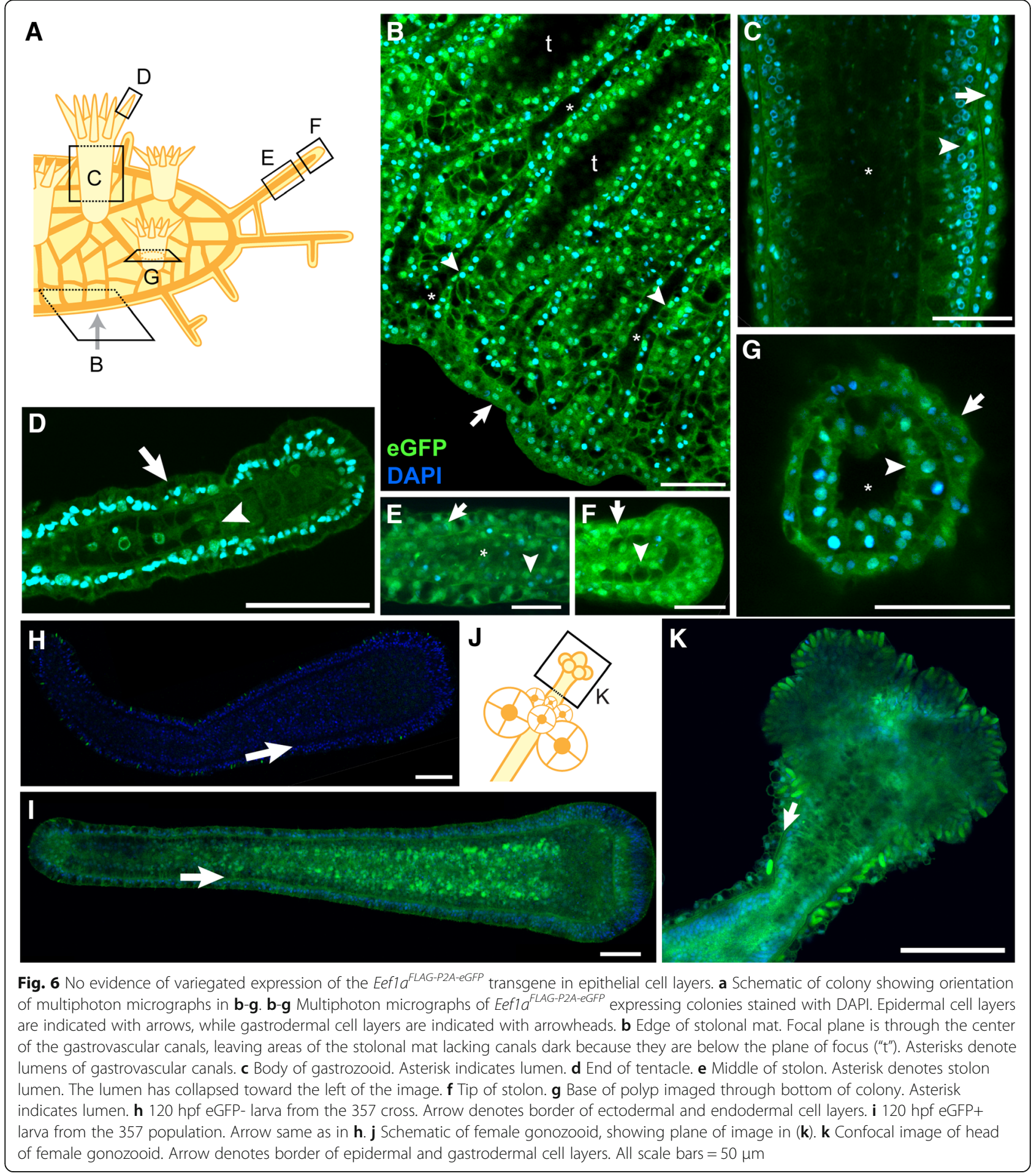

\section{Discussion}

Our study demonstrates that it is possible target the insertion of exogenous DNA into the Hydractinia genome via CRISPR/Cas9 genome editing. Specifically, we inserted either a FLAG-P2A-eGFP or a SF-P2A-tdTomato coding sequence into the Eef1a locus. With moderate effort, we were able to generate mosaic founders that transmitted each transgene through the germline, thus generating fully transgenic animals. We then used these animals to confirm the correct insertion of the reporter construct. Transgene expression in these offspring was stable throughout the duration of our experiments and across two generations of breeding. With this work, Hydractinia becomes the first cnidarian 


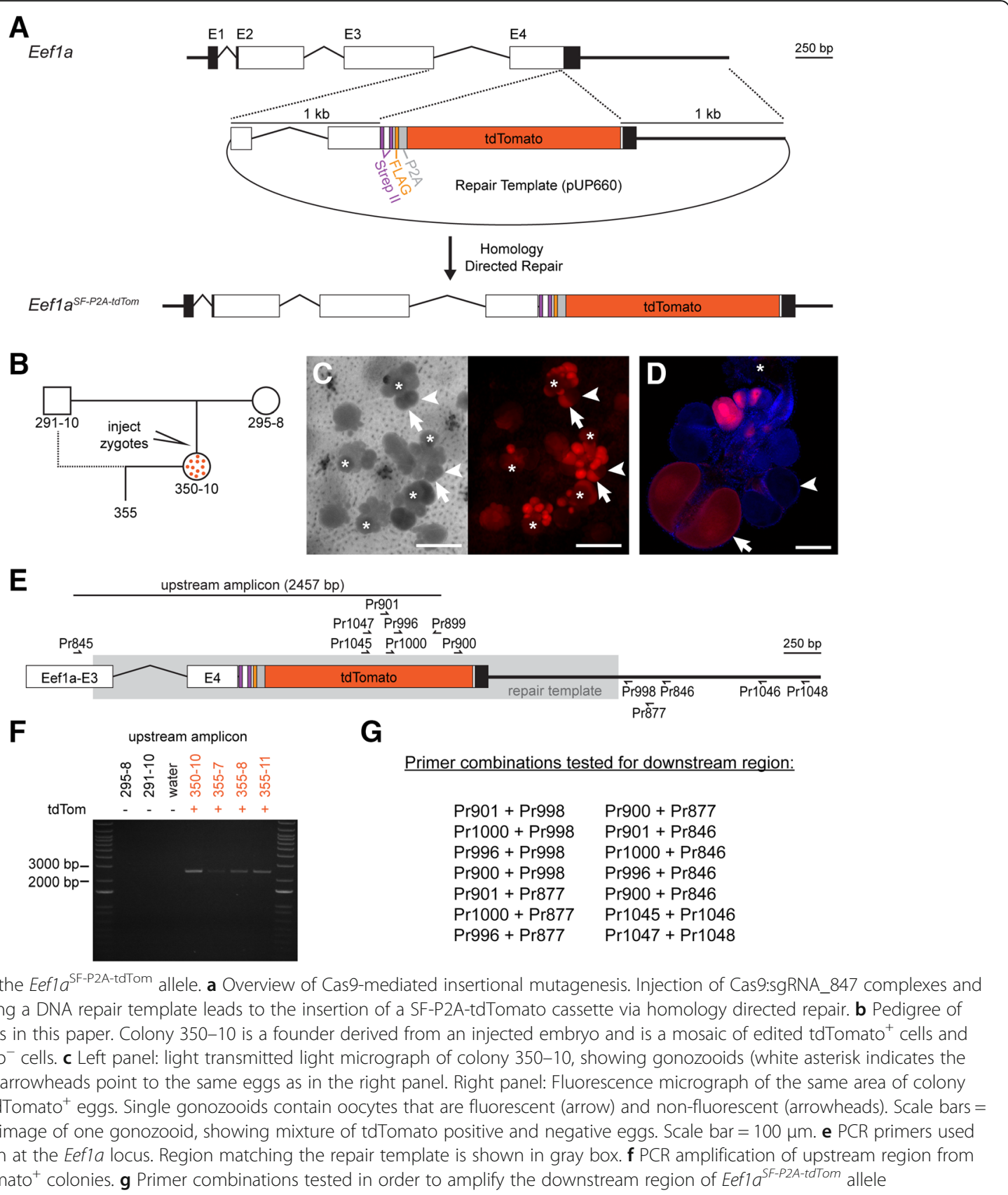

to display successful germline transmission of CRISPR/ Cas9 generated transgenes and, along with Nematostella [40], only the second cnidarian in which CRISPR/Cas9 mediated homologous recombination has been achieved.

In both cases of germline transmission in Hydractinia, we observed that the genomic regions upstream of the inserted transgene matched the expected sequence, but downstream regions did not. Specifically, we discovered a 681 bp deletion in the Eef1a $a^{\text {FLAG-P2A-eGFP }}$ allele and were unable to amplify the downstream region of the Eef1a ${ }^{F L A G-P 2 A-t d T o m}$ allele. One interpretation of these results is that homology directed repair (HDR) in Hydractinia could be more error-prone than anticipated. Specifically, our results are consistent with a scenario in which the lesion created by Cas9 results in significant alterations to both up- and downstream regions, which are then repaired by HDR or another form of DNA repair. Since we used fluorescence to screen for founder colonies, we were only able to detect transgenic animals in which the upstream regions preserved the reading frame of the reporter. Although the presence of downstream mutations did not create a problem in this study, 


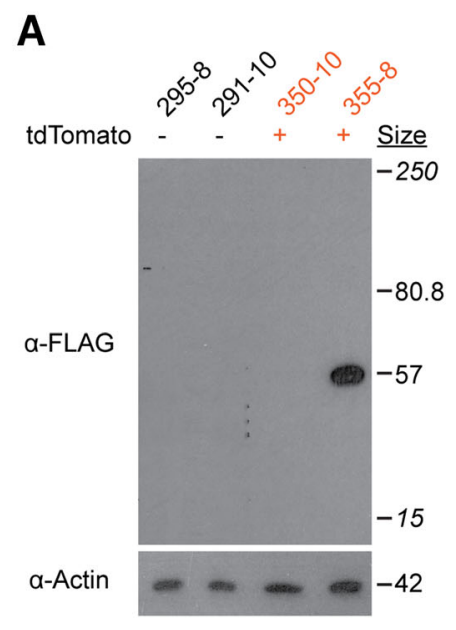

C

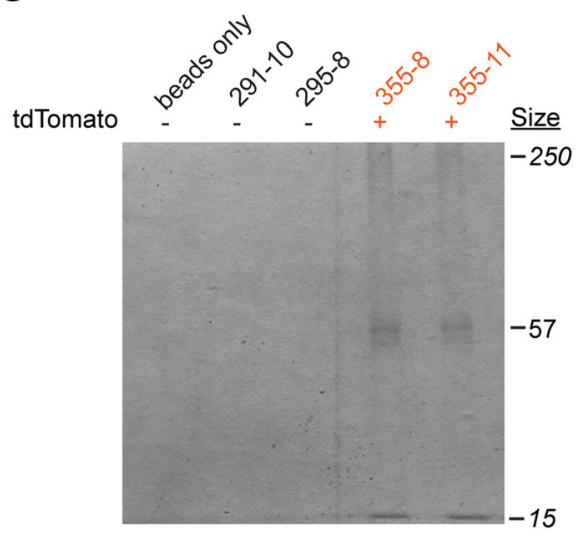

B

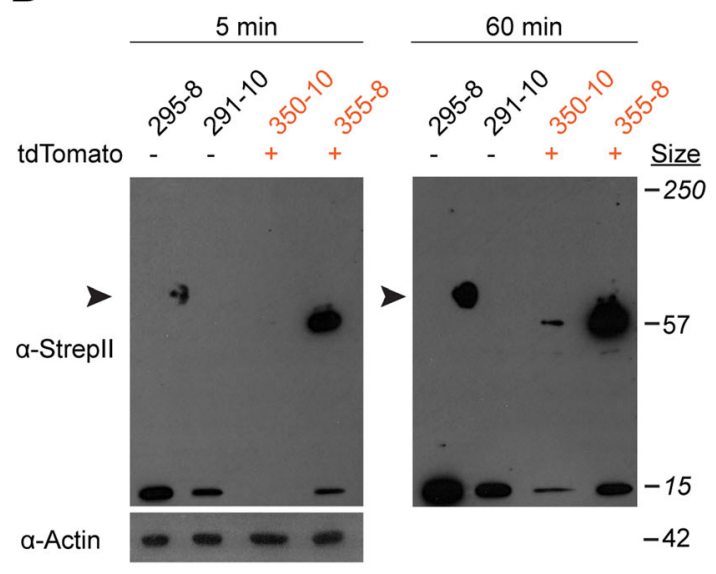

D

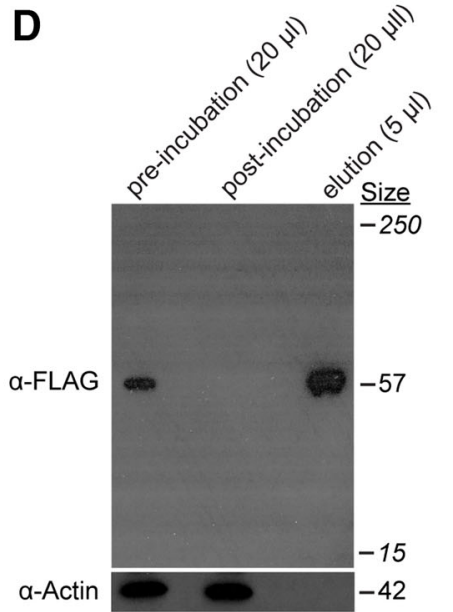

Fig. 8 Use of the Strepll-FLAG tag in immunoblots and pull-downs. a Western blot for FLAG on total protein lysates from Eef1a ${ }^{\text {FLAG-P2A-tdTom }}$ transgenic and nontransgenic colonies. Sizes in italics are the top and bottom bands of the molecular size marker. $\mathbf{b}$ Western blot of Strepll tag on total protein lysates from the same colonies. The blot was treated to induce chemiluminescence and exposed to film for 5 min (left), followed by a second exposure for $60 \mathrm{~min}$ (right). The $15 \mathrm{kDa}$ band suggests this antibody cross-reacts with some variants of an endogenous Hydractinia protein. Arrowhead indicates non-specific staining of protein ladder. c Coomassie stained SDS-PAGE gel of elutions from Strep-Tactin coated beads following incubation in total protein lysates from the indicated colonies. $\mathbf{d}$ Western blot against the FLAG peptide on the indicated amount of total protein lysate from colony $355-8$, before and after incubation with the anti-streptavidin beads. The elution from the beads was run alongside for comparison

it could complicate future work that depends on the sequence on both sides of an inserted transgene. It will therefore be important to determine the conditions that promote and/or prevent these mutations.

One variable that could affect the prevalence of mutations in surrounding regions is the size of the homology arms in the repair template. In this study, we followed the example of Ikmi et al. [40] and used $1 \mathrm{~kb}$ homology arms. However, homology arms of $\sim 500$ bp are routinely used to insert $1 \mathrm{~kb}$ transgenes in mammalian cells, while shorter transgenes can be inserted using homology arms as short as $40 \mathrm{bp}$ and a single stranded oligoDNA template [48]. Shortening the homology arms in Hydractinia might narrow the region available for homologous recombination and thus decrease the odds of recovering animals with large mutations. Further investigating the effect of homology arm length on the efficiency and fidelity of CRISPR/Cas9 mediated HDR in Hydractinia will be useful.

Our results demonstrate the feasibility of using short affinity tags to track endogenous proteins in vivo. One immediate application is to tag proteins in order to study their expression via immunolocalization or western blot. We showed here that the FLAG octopeptide is suitable for both applications in Hydractinia. This approach could, in some instances, eliminate the need to generate and validate antibodies in order to study a protein of interest. Another potential application is the purification of in vivo 
expressed proteins to study biochemical features such as post-translational modifications or interaction partners. Using relatively small amounts of tissue (area $\sim 6 \mathrm{~cm}^{2}$ ) and a simple incubation procedure with Strep-Tactin beads, we were able to extract all detectable StrepII-tagged Eef1a protein from total cell lysates in sufficient amounts to visualize on a coomassie stained polyacrylamide gel. One caveat to this result is that Eef1a is typically one of the most abundant proteins in eukaryotic cells, making it an easy target. Nonetheless, we expect that purifying less abundant proteins will be as simple as increasing the amount of starting tissue, a straightforward task in a clonally reproducing animal like Hydractinia. In short, this approach should enable Hydractinia researchers to study previously intractable questions regarding protein biochemistry using commercially available reagents.

To the best of our ability, we have sought to determine whether every cell in a transgenic colony is marked with a fluorescent reporter. In no instance did we see evidence of cells lacking expression of either eGFP or tdTomato when it is placed under control of the endogenous Eefla promoter. This suggests that Eefla will be a useful landing site for the ubiquitous expression of transgenes in future experiments. In addition, animals we generated in this study will be useful in experiments in which it is desirable to track cells over long periods of time in live colonies. Specifically, we expect to be able to use them to track cell migrations following the fusion of colonies with different degrees of histocompatibility. Such experiments will enable us to test previous claims regarding the cell types that migrate within chimeras, the speed and mode by which they migrate, and their ultimate fate $[36,49,50]$.

While our study is a significant advancement for the cnidarian research community, there are several limitations that should be considered. First, we have only demonstrated successful editing at one gene, Eefla. Complications may arise when targeting other loci. Second, we have made little attempt to optimize the microinjection cocktail. Our efficiency might be increased by altering the overall concentrations and/or ratios of donor template, Cas 9 protein, and sgRNA. Third, we cannot rule out the possibility that the repair template has been incorporated in the Hydractinia genome outside Eef1a. In fact, given the demonstrated ease with which exogenous DNA incorporates at random into the Hydractinia genome, we suspect other integrations did occur but were not detectable because our transgene was designed to express a functional protein only when integrated into an existing gene in the proper reading frame. Random integrations, if they were to disrupt an endogenous gene, could alter the phenotype of a transgenic colony. Researchers using CRISPR/Cas9 genome editing to insert transgenes in the future should therefore confirm any link between an observed phenotype and the mutant genotype either by creating knockins in different genetic backgrounds, breeding to confirm that phenotypes co-segregate with the transgene, and/or probing the genomes of transgenic colonies via Southern blots or whole genome resequencing. A fourth limitation is that we observed an obvious deleterious effect in colonies expressing Eef1 $a^{S F-P 2 A-t d T o m}$, but we did not determine whether this was due to toxicity of the tdTomato protein, the fusion of the SF-TAP tag to Eefla, the SF-TAP tag itself, the potential deletion of the region downstream of Eef1a, or some combination of these factors. Testing these possibilities in future work will be straightforward. Finally, we were unable to immunoprecipitate Eef1a-FLAG in these studies. It is likely that insertion of a 3X FLAG tag in the Eef1a locus (instead of the $1 \mathrm{X}$ tag used in this study) would have allowed for anti-FLAG immunoprecipitation.

Beyond this work, the ability to use CRISPR/Cas9 genome editing to create targeted knockins should enable the Hydractinia community to study other genes of interest. It also suggests a more efficient way to screen for gene knockouts because one could design a gene trap with a fluorescent reporter, thus enabling visual screening for mutants, and germline transmitted mutations, rather than laboriously genotyping by PCR. In addition, it should now be possible to begin developing more sophisticated functional genetic tools in Hydractinia, including the use of systems such as Cre/lox to create inducible or conditional expression or deletion systems.

For decades, Hydractinia has been studied by a small but dedicated community of scientists asking fundamental biological questions. Are Hydractinia stem cells truly pluripotent? Are there limits to the regenerative potential of this species? How does the division of labor and polyp polymorphism evolve in colonial species? What controls colony form during ontogeny? What allows a Hydractinia colony to distinguish itself from other colonies so accurately? Do colonies age? We expect that the availability of high throughput genomics and genome editing will speed us toward the answers. Along the way, we hope that established cnidarian researchers and newcomers alike will join the Hydractinia community to ask novel questions and enrich our understanding of animal biology.

\section{Conclusion}

This work is the first account of CRISPR/Cas9 mediated knockins in Hydractinia and the first example of the germline transmission of a CRISPR/Cas9 inserted transgene in a cnidarian. We expect this technology, when combined with high quality genomic resources and the logistical feasibility of genetic studies, will make 
Hydractinia a widely used model system for functional genomics.

\section{Methods}

Animal care and breeding

$H$. symbiolongicarpus was maintained at the University of Pittsburgh. Colonies were grown on glass microscope slides and cultured in 38 liter aquaria filled with artificial seawater (Instant Ocean Reef Crystals) between 28 and 31 ppt and maintained at 22-23 ${ }^{\circ} \mathrm{C}$. Adult colonies were fed 3-day-old Artemia nauplii (Brine Shrimp Direct) on Monday, Wednesday, and Friday. On Tuesday and Thursday, colonies were fed a suspension of pureed oysters (fresh caught, shucked, pureed, then stored frozen). Breeding colonies were kept on a day/night cycle of $8 \mathrm{~h}$ light, $16 \mathrm{~h}$ dark. Following their first exposure to light, male and female colonies were placed in separate 3 liter bins, where they released sperm or eggs 1$1.5 \mathrm{~h}$ later. Within $20 \mathrm{~min}$ of spawning, eggs were harvested by filtering water from the female bin through a $20 \mu \mathrm{m}$ cell strainer, and sperm was harvested by collecting $10-15 \mathrm{ml}$ from the male container. Sperm and eggs were mixed together with an additional $15 \mathrm{ml}$ artificial seawater in a $100 \mathrm{~mm}$ polystyrene petri dish, where fertilization occurred. For crosses not involving microinjection, embryos were allowed to develop into planula larvae and were settled 72-96 h post fertilization (hpf). Metamorphosis was induced by incubating the larvae in $56 \mathrm{mM} \mathrm{CsCl}$ in filtered seawater for $4-6 \mathrm{~h}$, and then transferring them with glass pipettes onto $25 \times 75 \mathrm{~mm}$ glass microscope slides. Three days later, larvae that successfully metamorphosed primary polyps were hand-fed pieces of 3-day-old Artemia nauplii. Hand feeding continued daily until polyps were able to catch and eat whole Artemia nauplii on their own, whereupon they were fed identically to the adult colonies as detailed above.

\section{Identification and cloning of Eef1a}

Previously published $H$. symbiolongicarpus RNA-Seq data [45] were used to identify the Eef1a sequence. The Eef1a genomic sequence was identified by BLAST searches against a draft genome of 291-10 [30]. BLAST databases were created and searched using using BLAST +2.4 [51]. The genomic region encoding exons 3-4 of the 295-8 Eef1a alleles (Additional file 2) were PCR-amplified using primers P845 and Pr846 (Additional file 1: Table S1) and Phusion High-Fidelity DNA Polymerase (ThermoFisher F530). PCR products were gel purified using the Qiagen Gel Extraction Kit (Qiagen 28,706), cloned using the Blunt II TOPO Cloning Kit (Invitrogen K2800), and then sequenced.

\section{Genomic DNA extraction}

Colonies were starved for at least 2 days before to DNA extraction. Tissue was harvested by scraping it from slide with a razor blade and transferring it into a $1.5 \mathrm{ml}$ microcentrifuge tube, briefly centrifuging at max speed $(>21,000 \mathrm{~g})$, pipetting off the seawater, and storing at $80{ }^{\circ} \mathrm{C}$ until extraction. To extract genomic DNA, UEB1 buffer $(7 \mathrm{M}$ urea, $0.3125 \mathrm{M} \mathrm{NaCl}, 0.05 \mathrm{M}$ Tris- $\mathrm{HCl}$, $0.02 \mathrm{M}$ EDTA, and 1\% w:v N-lauroylsarcosine sodium salt) was added to the tissue, which was then ground with a pestle and incubated at $37^{\circ} \mathrm{C}$ for $1 \mathrm{~h}$. This was followed by a phenol/chloroform extraction and precipitation with 2.5 volumes $100 \%$ ethanol and $1 / 10$ volume $5 \mathrm{M}$ sodium acetate ( $\mathrm{pH}$ 5.2). The precipitate was pelleted, washed with $70 \%$ ethanol, and resuspended with TE. RNA was removed by adding 1/100th volume of Ambion RNAse cocktail (Ambion \#AM2286) and incubating at $37{ }^{\circ} \mathrm{C}$ for $15 \mathrm{~min}$. This was followed by another ethanol precipitation. The precipitate was pelleted, washed with $70 \% \mathrm{EtOH}$, resuspended with $\mathrm{TE}$, and diluted to a working stock concentration of $25 \mathrm{ng} / \mu \mathrm{l}$.

\section{Eef1a CRISPR target sequence selection}

Before designing the repair template plasmid, a CRISPR target sequence needed to be selected. The genomic sequence of all four Eef1a alleles spanning primers Pr845 and Pr846 were aligned using Clustal $\Omega$ [52]. From this alignment (Additional file 3), the 3'-most PAM motif prior to the stop codon was identified and the preceding 20 nucleotides were selected as the CRISPR target sequence (sgRNA_847; Fig. 1c and Additional file 1: Table S1). To check for any obvious off-target potential, sgRNA_847 was blasted against the previously described BLAST database of the 291-10 draft genome. BLAST alignments were manually inspected for significant matches ( $>15$ consecutive matching bases, starting at the first base prior the start of the PAM motif). Aside from the two 291-10 Eefla alleles, no significant matches were found elsewhere in the genome.

\section{Construct design and synthesis}

The Eefla-FLAG-P2A-eGFP donor plasmid was assembled using the NEBuilder HiFi DNA Assembly kit (NEB E5520). The upstream and downstream homology arms were amplified by PCR from colony 291-10 using primers P845 and Pr846 (Additional file 1: Table S1). The Hydractinia codon optimized eGFP sequence was PCR amplified from pAct-I:51Act-eGFP [36]. Nucleotide sequences of the FLAG tag and P2A self cleaving peptide [53] were first codon optimized using a codon usage table generate from a publicly available $H$. symbiolongicarpus transcriptome [45] and synthesized as oligoDNA primers to be incorporated into the plasmid during Gibson assembly. The Eef1a-SF-P2A-tdTomato donor plasmid was assembled in 
a similar fashion. The SF-TAP sequence [46] was codon optimized and synthesized as an oligoDNA primer for assembly while a Hydractinia codon-optimized tdTomato sequence was amplified from a plasmid obtained from the Frank lab. These sequences were assembled and cloned using the NEBuilder HiFi DNA Assembly kit and sequenced to validate the assembly (Additional file 4). Plasmid DNA for microinjection was prepared using the GeneJET Plasmid Midiprep kit (ThermoFisher K0481), followed by an additional phenol/chloroform purification, ethanol/sodium acetate precipitation, and resuspension in $1 \mathrm{X} \mathrm{TE}$ at a concentration of $1 \mu \mathrm{g} / \mu \mathrm{l}$. The plasmid was then diluted with a working buffer (20 mM HEPES; $150 \mathrm{mM} \mathrm{KCl}, \mathrm{pH} \mathrm{7.5)}$ to a final concentration of $600 \mathrm{ng} /$ $\mu \mathrm{l}$, and stored at $-20^{\circ} \mathrm{C}$.

\section{Microinjection}

To prepare embryos for microinjection, a cross was performed as described above. After allowing fertilization to take place in the $100 \mathrm{~mm}$ dish for $15 \mathrm{~min}$, the fertilized eggs were washed three times with filtered seawater in a $50 \mathrm{ml}$ conical tube to remove excess sperm and remnants of the jelly coat. Washed embryos were then loaded onto a glass microscope slide and injected until the first cell cleavage began.

The microinjection cocktail was prepared prior to injection. Recombinant Cas9 protein (PNA Bio) and a synthetic sgRNA (Synthego) were combined and incubated at $37{ }^{\circ} \mathrm{C}$ for $10 \mathrm{~min}$, after which the donor plasmid was added. The final injection cocktail consisted of $1 \mu \mathrm{g} / \mu \mathrm{l}$ Cas $9,500 \mathrm{ng} / \mu \mathrm{l} \mathrm{sgRNA}$, and $200 \mathrm{ng} / \mu \mathrm{l}$ donor plasmid. Immediately before injection, the cocktail was centrifuged at max speed $(>21,000 \mathrm{~g}$ ) for approx. 5 min to pellet precipitates and particles that would clog the injection needle. The supernatant was then loaded into the microinjection needle and injected into single-cell Hydractinia embryos using a FemtoJet (Eppendorf) microinjection system.

\section{Tissue fixation and Immunolocalization}

To prepare fixed tissue, animals were anaesthetized with menthol crystals (Sigma M2772) or 2\% urethane in seawater, followed by fixation in $4 \%$ paraformaldehyde in seawater for $1 \mathrm{~h}$ at room temperature (RT) or overnight $(\mathrm{ON})$ at $4{ }^{\circ} \mathrm{C}$. Tissue was permeabilized by washing three times with PBS $+0.3 \%$ Triton X-100 (PBST) for $30 \mathrm{~min}$ at $4{ }^{\circ} \mathrm{C}$. For immunolocalizations, all steps were carried out at RT unless stated otherwise. Following fixation tissues were then blocked in 3\% BSA/PBST (two incubations, $30 \mathrm{~min}$ each), incubated $\mathrm{ON}$ at $4{ }^{\circ} \mathrm{C}$ with $1: 1000$ dilution of mouse anti-FLAG primary antibody (Sigma F1804) in 3\% BSA/PBST, washed with PBST (three washes, $10 \mathrm{~min}$ each), blocked in $3 \% \mathrm{BSA} / 5 \%$ goat serum/PBST for $30 \mathrm{~min}$, incubated for $1 \mathrm{~h}$ with a 1:1000 dilution of an Alexa 647 conjugated goat anti-mouse secondary antibody (ThermoFisher A-21235) in 3\% BSA $/ 5 \%$ goat serum/PBST, and washed with PBST (three washes, $10 \mathrm{~min}$ each). Nuclei were then stained $\mathrm{ON}$ at $4{ }^{\circ} \mathrm{C}$ with $5 \mu \mathrm{g} / \mathrm{ml}$ DAPI, washed with PBST (three washes at $4{ }^{\circ} \mathrm{C}, 30 \mathrm{~min}$ each), and mounted in Gelvatol mounting medium [54] prior to imaging.

\section{Microscopy}

Images of live colonies were collected on an Olympus macroscope fitted with a $1 \mathrm{X}$ objective equipped with a Retiga camera and controlled by Nikon NIS Elements software. Three dimensional images of larvae collected using a Nikon A1 confocal microscope in galvanometer scanning mode with an ELWD 40x water objective (NA 1.12) with Nyquist sampling in $\mathrm{X}, \mathrm{Y}$ and $\mathrm{Z}$. Images were rendered and stitched using NIS elements. Confocal images of other tissues were acquired on a Nikon A1 scanning confocal microscope with 20X air objectives (NA 0.75). For multiphoton microscopy, we used a Nikon A1R MP microscope configured with the Nikon Ni-E (Nikon Instruments, Tokyo, Japan) upright motorized system. The microscope was equipped with a Chameleon Laser Vision (Coherent, Inc., CA, USA) tuned to $850 \mathrm{~nm}$ for GFP excitation, an APO LWD 25× water immersion objective with 1.1 NA, a high-speed (30 frames/s) resonant scanning mode and a galvano scanning mode suitable for linescanning acquisitions. The Nano-Drive system was used to acquire fine high speed control of z-plane selection. The fluorescence detection unit of the microscope consisted of four detectors (photo multiplying tubes). We used detector 2 for eGFP 525/50 nm (green channel). The microscope components including the laser, stage, resonant scanning head, detectors and acquisition were controlled using NIS Elements software (Nikon). Post-processing was carried out in NIS Elements software (v. 4.5) and exported as TIFF files. All adjustments were applied equally to the entire image.

\section{Colony area estimation}

Colony area estimated by visualizing live colonies with a Leica M80 stereoscope at 10X magnification and acquiring color images in JPEG format with a Leica IC80HD camera. Images were imported into ImageJ as implemented in Fiji [55]. This was converted into a binary black and white image by adjusting the threshold level until all pixels within the colony perimeter were black and the background remained white. The "Analyzed Particles" command was then used to compute the area of the colony. The scale was determined by imaging a ruler at the same magnification. Statistical analysis was performed in GraphPad Prism. 


\section{Genotyping}

Genomic inserts were amplified using the Q5 High-Fidelity 2X Master Mix (NEB catalog M0492). To amplify each junction, PCR primers were designed so that one of the primers is tethered to the endogenous gDNA sequence of the EF1- $\alpha$ locus and the paired primer to the insert (Figs. 3 and 7). For a $50 \mu \mathrm{L}$ PCR, 100 ng of gDNA was used. PCRs were analyzed by electrophoresis on $1 \%$ agarose gels. PCR products were purified using the GeneJET PCR Purification Kit (ThermoFisher K0701), cloned using the Blunt II TOPO Cloning Kit (Invitrogen K2800), and then sequenced.

\section{Protein extraction}

Total protein was extracted from whole colonies of $H$. symbiolongicarpus. Prior to tissue collection, a lysis buffer consisting of RIPA buffer (Sigma R0278), $1 \mathrm{mM}$ AEBSF, $1 \mu \mathrm{g} / \mathrm{ml}$ pepstatin A, $5 \mathrm{mM}$ EDTA pH 8, $1 \mathrm{mM}$ EGTA pH 8, and 1X cOmplete ${ }^{\mathrm{Tm}}$ EDTA-free protease inhibitor cocktail tabs (Roche \#11836170001) was prepared. Colonies were excised from glass slides, collected in eppendorf tubes, and briefly centrifuged at max speed $(>21,000 \mathrm{~g})$ to allow excess seawater to be removed. Then the lysis buffer was added and the tissue ground with a pestle and incubated on ice for 10-20 min. Lysates were then centrifuged at max speed $(>21,000 \mathrm{~g})$ for $10 \mathrm{~min}$ at $4{ }^{\circ} \mathrm{C}$. Lysate was decanted into a new tube and placed on ice. Protein was quantified using the Pierce BCA Protein Assay Kit (ThermoFisher 23,225), diluted with $4 \times$ SDS-PAGE loading buffer (with either 2-mercaptoethanol or DTT), boiled for $5 \mathrm{~min}$ at $100{ }^{\circ} \mathrm{C}$, aliquoted, and stored at $-80{ }^{\circ} \mathrm{C}$.

\section{Immunoblotting}

For western blotting, a total of $10 \mu \mathrm{g}$ of protein was run on Novex 10\% Tris-Glycine mini gels (ThermoFisher XP00105BOX). Protein was transferred to a PVDF membrane (Bio-Rad 1620177) using the Pierce G2 Fast Blotter. Blots were probed according to the supplier's recommended protocol for the following antibodies: anti-actin (Sigma A2066), anti-FLAG (Sigma F1804), and anti-StrepII (MBL International catalog \#M211-3). Secondary antibodies (anti-mouse IgG-peroxidase and anti-rabbit IgGperoxidase; Sigma A0168 and A0545, respectively) were used at 1:10,000 dilution. Signal was detected using SuperSignal West Pico Chemiluminescent Substrate kit (ThermoFisher 34,078), exposed to Carestream Biomax XAR Film (Sigma 1,651,454), and developed using a Konica SRX-101A tabletop processor.

\section{Pull-down assays}

Immunoprecipitations (IPs) on FLAG-P2A-eGFP expressing colonies were performed using Anti-FLAG M2 Magnetic Beads (Sigma M8823) following the supplier's recommended protocol. For lysates from SF-P2A-tdTomato expressing colonies, pull-downs were performed using MagStrep "type3" XT magnetic beads (IBA 24090-002). Total protein was extracted as described above. Total volume of the lysate was approx. $1 \mathrm{ml}$, this was added to $1 \mu \mathrm{l}$ of equilibrated beads and incubated $\mathrm{ON}$ at $4{ }^{\circ} \mathrm{C}$ turning end-over-end. Lysate was removed and beads were washed three times at RT using $1 \mathrm{ml}$ of Strep-Tactin XT Wash Buffer (IBA 2-1003-100). After final wash, beads were moved into a clean eppendorf tube and eluted by incubating beads with $30 \mu \mathrm{l}$ Strep-Tactin XT Elution Buffer (IBA 2-1042-025) for $10 \mathrm{~min}$ at RT with periodic vortexing. Eluted protein was diluted with $4 \times$ SDS-PAGE loading buffer (with DTT), boiled for $5 \mathrm{~min}$ at $100{ }^{\circ} \mathrm{C}$, and stored at $-80^{\circ} \mathrm{C}$.

IPs and pull-downs were analyzed by both coomassie staining and immunoblotting (as described above). For coomassie staining, $35 \mu \mathrm{l}$ of the eluate was run on a Novex $10 \%$ Tris-Glycine mini gel. The gel was then fixed for $10 \mathrm{~min}$ (10\% acetic acid, 25\% IPA) at RT, stained ON at RT in a $0.3 \%$ solution of G250 Brilliant Blue coomassie (BioRad 161-0406), destained with 10\% acetic acid for approx. Two hours, and then imaged.

\section{Additional files}

Additional file 1: Figure S1. Pedigree of the colonies used to generate zygotes for microinjection. Figure S2. Effects and outcomes of microinjection. Figure S3. eGFP ${ }^{+}$vs. eGFPdim larvae. Figure S4. FLAG immunolocalization controls. Table S1. Oligonucleotide sequences used in this study. (PDF $2121 \mathrm{~kb}$ )

Additional file 2: Alignment of cloned Eefia sequences from female colony 295-8. Two PCR amplicons (sized $2.4 \mathrm{~kb}$ and $2.0 \mathrm{~kb}$ ) from the genomic region encoding the $3^{\prime}$ end of Eef1 a in colony $295-8$ were cloned, sequenced ( 3 clones each), and aligned. This revealed two alleles, with the major differences being a 52 bp and a 313 bp indel. Differences are shown in red boxes. Sequence features are shown on and above the top sequence of the alignment. Green, Eef1 a mRNA as determined by alignments to 29110 transcripts. Yellow, Eef1a coding sequence. (PDF 4523 kb)

Additional file 3: Alignment of Eefla alleles from colonies 291-10 and 295-8. Nucleotide alignment of the region encoding exons 3 and 4 of Eefla from the four alleles in 291-10 and 295-8. Differences are highlighted in red boxes. Sequence features are shown on and above the top sequence of the alignment. Green, mRNA as determined by alignments to 291-10 transcripts. Yellow, coding sequence. Blue, sgRNA_847 binding site. (PDF 4205 kb)

Additional file 4: Genbank formatted sequence of repair template pUP613 (Eef1a-FLAG-P2A-eGFP). (TXT 9 kb)

Additional file 5: Alignment of upstream and downstream integration sites from Eef1 $a^{F L A G-P 2 A-E G F P}$ colonies. Nucleotide alignment of the regions encoding the up- and downstream integration sites from the founder colony, 347-10, and two transgenic offspring, 354-3 and 354-5. Three cloned amplicons were sequenced for each animal at each site. Primer binding sites are indicated in green on the expected sequence. Differences are highlighted in red boxes. Note that the regions that do not overlap between the upstream and downstream sequences are shown as gaps at the beginning and end of the alignment. (PDF $4434 \mathrm{~kb}$ )

Additional file 6: Z-stack of Eef1 $a^{F L A G-P 2 A-E G F P}$ gastrozooid. Only DAPI (blue) and eGFP (green) channels are shown. (MP4 8810 kb) 
Additional file 7: Z-stack of Eef1 $a^{\text {FLAG-P2A-GFP }}$ gastrozooid stained with anti-FLAG antibodies. Same polyp as in Additional file 6. Signal from Alexa 647 (white) is shown. (MP4 $5787 \mathrm{~kb}$ )

Additional file 8: Z-stack of non-transgenic gastrozooid. Only DAPI (blue) and eGFP (green) channels are shown. (MP4 4899 kb)

Additional file 9: Z-stack of non-transgenic gastrozooid stained with anti-FLAG antibodies. Same polyp as in Additional file 8. Signal from Alexa 647 (white) is shown. (MP4 $2059 \mathrm{~kb}$ )

Additional file 10: Z-stack of tentacle from Eef1a ${ }^{\text {FLAG-P2A-eGFP }}$ colony. (MP4 $8378 \mathrm{~kb}$ )

Additional file 11: Z-stack of gastrozooid from Eef1a $a^{\text {FLG-P2A-GFP }}$ colony. (MP4 $14636 \mathrm{~kb}$ )

Additional file 12: Z-stack of stolonal mat and base of gastrozooid from Eef1 ${ }^{\text {FLAG-P2A-EGFP }}$ colony. (MP4 $15146 \mathrm{~kb}$ )

Additional file 13: Z-stack of edge of stolonal mat from Eef1a $a^{\text {FLAG-P2A- }}$ eGFP colony. (MP4 $17001 \mathrm{~kb}$ )

Additional file 14: Z-stack of stolon from Eef1a ${ }^{\text {FLAGP2A-EFP }}$ colony. (MP4 $15225 \mathrm{~kb})$

Additional file 15: Z-stack of female gonozooid head from Eef1a $a^{F L A G-P 2 A-}$ eGPP colony. (MP4 $20004 \mathrm{~kb}$ )

Additional file 16: Z-stack of Eef1a $a^{\text {FLAG-P2A-GFP }}$ larva. (MP4 $9679 \mathrm{~kb}$ ) Additional file 17: Z-stack of non-transgenic larva. (MP4 $5615 \mathrm{~kb}$ ) Additional file 18: Genbank formatted sequence of repair template pUP660 (Eef1a-SF-P2A-tdTom) (TXT $10 \mathrm{~kb}$ )

Additional file 19: Alignment of upstream and downstream integration sites from Eef1 a ${ }^{\text {SF-P2A-tdTom }}$ colonies. Nucleotide alignment of the region encoding the upstream integration site from the founder colony, 350-10, and one transgenic offspring, 355-8. Three cloned amplicons were sequenced for each animal at each site. Primer binding sites are indicated in green on the expected sequence. (PDF $3670 \mathrm{~kb}$ )

\section{Abbreviations}

Eef1a: Eukaryotic elongation factor 1 alpha; IPs: Immunoprecipitations; ON: Overnight; PCR: Polymerase chain reaction; RT: Room temperature; SFTAP: Strepll-FLAG tandem affinity purification; sgRNA: Single guide RNA; UTR: Untranslated region

\section{Acknowledgements}

We thank Leo Buss from Yale University for providing inbred and field-collected colonies, Sebastien Gingras from the University of Pittsburgh Transgenic and Gene Targeting Core for helpful discussions and advice, and Simon Watkins of the Center for Biological Imaging at the University of Pittsburgh for assistance with imaging. This research was supported in part by the Intramural Research Program of the National Human Genome Research Institute, National Institutes of Health.

\section{Funding}

This work was supported by NSF grant IOS1557339 and startup funds from the Thomas E. Starzl Transplantation Institute to MLN, Science Foundation Ireland Principal Investigator award 11/PI/1020 to UF, and NIH grant ZIA-HG000140 to ADB. SMS was supported by NIH grant T32AI074490. The funding bodies played no role in the design, analysis, or interpretation of the data or in writing the manuscript.

\section{Availability of data and materials}

Genomic contigs containing 291-10 Eef1a alleles are available at NCBI. The draft Hydractinia genome assembly is freely available through the Hydractinia Genome Project Portal, at https://research.nhgri.nih.gov/hydractinia/. All other data generated and analysed during this study are included in this publication (including supplemental information).

\section{Authors' contributions}

SMS and MLN conceptualized and designed the experiments. SMS performed all experiments listed in the manuscript. ZM, JMH, and BMR were responsible for animal care and maintenance. HF duplicated results in UF's lab. SMS, MLN, GAG, and AMW performed imaging. CES and ADB provided
Hydractinia draft genome assemblies. MLN and SMS wrote the manuscript. All authors read and approved the final manuscript.

Ethics approval and consent to participate

Not applicable.

\section{Consent for publication}

Not applicable.

\section{Competing interests}

The authors declare that they have no competing interests.

\section{Publisher's Note}

Springer Nature remains neutral with regard to jurisdictional claims in published maps and institutional affiliations.

\section{Author details}

'Department of Surgery, Thomas E. Starzl Transplantation Institute, University of Pittsburgh, Pittsburgh, PA, USA. ${ }^{2}$ Pittsburgh Center for Evolutionary Biology and Medicine, University of Pittsburgh, Pittsburgh, PA, USA. ${ }^{3}$ Center for Biological Imaging and Department of Cell Biology, University of Pittsburgh, Pittsburgh, PA, USA. ${ }^{4}$ Centre for Chromosome Biology, School of Natural Sciences, National University of Ireland, Galway, Ireland. ${ }^{5}$ Whitney Laboratory for Marine Bioscience, and Department of Biology, University of Florida, St. Augustine, FL, USA. ${ }^{6}$ Computational and Statistical Genomics Branch, National Human Genome Research Institute, National Institutes of Health, Bethesda, MD, USA. ${ }^{7}$ Department of Immunology, University of Pittsburgh, Pittsburgh, PA, USA.

Received: 15 June 2018 Accepted: 22 August 2018

Published online: 03 September 2018

\section{References}

1. Steele RE, David CN, Technau U. A genomic view of 500 million years of cnidarian evolution. Trends Genet. 2011:27:7-13.

2. Technau U, Steele RE. Evolutionary crossroads in developmental biology: Cnidaria. Development. 2011;138:1447-58.

3. Davy SK, Allemand D, Weis VM. Cell biology of cnidarian-dinoflagellate symbiosis. Microbiol Mol Biol Rev. 2012;76:229-61.

4. Rosengarten RD, Nicotra ML. Model systems of invertebrate allorecognition. Curr Biol. 2011;21:R82-92.

5. Juliano CE, Reich A, Liu N, Götzfried J, Zhong M, Uman S, et al. PIW proteins and PIWI-interacting RNAs function in Hydra somatic stem cells. Proc Natl Acad Sci U S A. 2014;111:337-42.

6. Gahan JM, Bradshaw B, Flici H, Frank U. The interstitial stem cells in Hydractinia and their role in regeneration. Curr Opin Genet Dev. 2016; 40:65-73

7. Layden MJ, Rentzsch F, Röttinger E. The rise of the starlet sea anemone Nematostella vectensis as a model system to investigate development and regeneration: Overview of starlet sea anemone Nematostella vectensis. WIREs Dev Biol. 2016:5:408-28.

8. Bosch TCG, Klimovich A, Domazet-Lošo T, Gründer S, Holstein TW, Jékely G, et al. Back to the Basics: Cnidarians Start to Fire. Trends Neurosci. 2017:40: 92-105

9. Kelava I, Rentzsch F, Technau U. Evolution of eumetazoan nervous systems: insights from cnidarians. Philos Trans R Soc Lond B Biol Sci. 2015;370: 20150065

10. Carter JA, Hyland C, Steele RE, E-MS C. Dynamics of Mouth Opening in Hydra. Biophys J. 2016;110:1191-201.

11. Cochet-Escartin O, Locke TT, Shi WH, Steele RE, E-MS C. Physical Mechanisms Driving Cell Sorting in Hydra. Biophys J. 2017;113:2827-41.

12. Schenkelaars Q, Tomczyk S, Wenger Y, Ekundayo K, Girard V, Buzgariu W, et al. Hydra, a model system for deciphering the mechanisms of aging and resistance to aging. bioRxiv. 2017;155804.

13. Bellantuono AJ, Bridge D, Martínez DE. Hydra as a tractable, long-lived model system for senescence. Invertebr Reprod Dev. 2015:59:39-44.

14. Pisani D, Pett W, Dohrmann M, Feuda R, Rota-Stabelli O, Philippe $H$, et al. Genomic data do not support comb jellies as the sister group to all other animals. Proc Natl Acad Sci U S A. 2015;112:15402-7. 
15. Whelan NV, Kocot KM, Moroz TP, Mukherjee K, Williams P, Paulay G, et al. Ctenophore relationships and their placement as the sister group to all other animals. Nat Ecol Evol. 2017;1:1737-46.

16. Putnam NH, Srivastava M, Hellsten U, Dirks B, Chapman J, Salamov A, et al. Sea anemone genome reveals ancestral eumetazoan gene repertoire and genomic organization. Science. 2007;317:86-94.

17. Kortschak RD, Samuel G, Saint R, Miller DJ. EST analysis of the cnidarian Acropora millepora reveals extensive gene loss and rapid sequence divergence in the model invertebrates. Curr Biol. 2003;13:2190-5.

18. Maxwell EK, Schnitzler CE, Havlak P, Putnam NH, Nguyen AD, Moreland R, et al. Evolutionary profiling reveals the heterogeneous origins of classes of human disease genes: implications for modeling disease genetics in animals. BMC Evol Biol. 2014;14:212.

19. Schierwater B, Murtha M, Dick M, Ruddle FH, Buss LW. Homeoboxes in cnidarians. J Exp Zool. 1991;260:413-6.

20. Cartwright P, Bowsher J, Buss LW. Expression of a Hox gene, Cnox-2, and the division of labor in a colonial hydroid. Proc Natl Acad Sci U S A. 1999;96: 2183-6

21. Duffy DJ, Plickert G, Kuenzel T, Tilmann W, Frank U. Wnt signaling promotes oral but suppresses aboral structures in Hydractinia metamorphosis and regeneration. Development. 2010;137:3057-66.

22. Bradshaw B, Thompson K, Frank U. Distinct mechanisms underlie oral vs aboral regeneration in the cnidarian Hydractinia echinata. Elife. 2015;4: e05506

23. Cartwright $P$, Collins A. Fossils and phylogenies: integrating multiple lines of evidence to investigate the origin of early major metazoan lineages. Integr Comp Biol. 2007:47:744-51.

24. Peterson KJ, Cotton JA, Gehling JG, Pisani D. The Ediacaran emergence of bilaterians: congruence between the genetic and the geological fossil records. Philos Trans R Soc Lond B Biol Sci. 2008;363:1435-43.

25. dos Reis M, Thawornwattana Y, Angelis K, Telford MJ, Donoghue PCJ, Yang Z. Uncertainty in the Timing of Origin of Animals and the Limits of Precision in Molecular Timescales. Curr Biol. 2015;25:2939-50.

26. Schwentner M, Bosch TCG. Revisiting the age, evolutionary history and species level diversity of the genus Hydra (Cnidaria: Hydrozoa). Mol Phylogenet Evol. 2015;91:41-55.

27. Kumar S, Stecher G, Suleski M, Hedges SB. TimeTree: A Resource for Timelines, Timetrees, and Divergence Times. Mol Biol Evol. 2017:34:1812-9.

28. Cartwright $P$. The development and evolution of hydrozoan polyp and colony form. Hydrobiologia. 2004:530:309-17.

29. Cartwright $P$, Buss LW. Colony integration and the expression of the Hox gene, Cnox-2, in Hydractinia symbiolongicarpus (Cnidaria: Hydrozoa). J Exp Zool. 1999;285:57-62.

30. Schnitzler CE, Nguyen AD, Koren S, Gornik SD, Plickert G, Buss L, et al. The Genomics of Hydractinia: Understanding Regeneration, Allorecognition, and Stem Cell Biology. Integr Comp Biol. 2018;58:E203.

31. Nicotra ML, Powell AE, Rosengarten RD, Moreno M, Grimwood J, Lakkis FG, et al. A hypervariable invertebrate allodeterminant. Curr Biol. 2009;19:583-9.

32. Rosa SF, Powell AE, Rosengarten RD, Nicotra ML, Moreno MA, Grimwood J, et al. Hydractinia allodeterminant alr 1 resides in an immunoglobulin superfamily-like gene complex. Curr Biol. 2010;20:1122-7.

33. Duffy DJ, Millane RC, Frank U. A heat shock protein and Wnt signaling crosstalk during axial patterning and stem cell proliferation. Dev Biol. 2012; 362:271-81.

34. Millane RC, Kanska J, Duffy DJ, Seoighe C, Cunningham S, Plickert G, et al. Induced stem cell neoplasia in a cnidarian by ectopic expression of a POU domain transcription factor. Development. 2011;138:2429-39.

35. Kanska J, Frank U. New roles for Nanos in neural cell fate determination revealed by studies in a cnidarian. J Cell Sci. 2013;126:3192-203.

36. Kunzel T, Heiermann R, Frank U, Muller W, Tilmann W, Bause M, et al. Migration and differentiation potential of stem cells in the cnidarian Hydractinia analysed in eGFP-transgenic animals and chimeras. Dev Biol. 2010;348:120-9.

37. DuBuc TQ, Stephenson TB, Rock AQ, Martindale MQ. Hox and Wnt pattern the primary body axis of an anthozoan cnidarian before gastrulation. Nat Commun. 2018:9:2007.

38. Pukhlyakova E, Aman AJ, Elsayad K, Technau U. $\beta$-Catenin-dependent mechanotransduction dates back to the common ancestor of Cnidaria and Bilateria. Proc Natl Acad Sci U S A. 2018;115:6231-6236.

39. Servetnick MD, Steinworth B, Babonis LS, Simmons D, Salinas-Saavedra M, Martindale MQ. Cas9-mediated excision ofNematostella brachyurydisrupts endoderm development, pharynx formation and oral-aboral patterning. Development. 2017:144:2951-60.

40. Ikmi A, McKinney SA, Delventhal KM, Gibson MC. TALEN and CRISPR/Cas9mediated genome editing in the early-branching metazoan Nematostella vectensis. Nat Commun. 2014;5:5486.

41. Quiroga Artigas G, Lapébie P, Leclère L, Takeda N, Deguchi R, Jékely G, et al. A gonad-expressed opsin mediates light-induced spawning in the jellyfish Clytia. Elife. 2018;7:e29555.

42. Cleves PA, Strader ME, Bay LK, Pringle JR, Matz MV. CRISPR/Cas9-mediated genome editing in a reef-building coral. Proc Natl Acad Sci U S A. 2018;115: 5235-40.

43. Gahan JM, Schnitzler CE, DuBuc TQ, Doonan LB, Kanska J, Gornik SG, et al. Functional studies on the role of Notch signaling in Hydractinia development. Dev Biol. 2017;428:224-31.

44. Dupre C, Grasis JA, Steele RE, Schnitzler CE, Juliano CE. Hydroidfest 2016: celebrating a renaissance in hydrozoan research. Evodevo. 2017:8:7.

45. Sanders SM, Shcheglovitova M, Cartwright P. Differential gene expression between functionally specialized polyps of the colonial hydrozoan Hydractinia symbiolongicarpus (Phylum Cnidaria). BMC Genomics. 2014;15:406.

46. Gloeckner CJ, Boldt K, Ueffing M. Strep/FLAG tandem affinity purification (SF-TAP) to study protein interactions. Curr Protoc Protein Sci. Wiley Online Library. 2009;57:19.20.1-19.20.19.

47. Shaner NC, Campbell RE, Steinbach PA, Giepmans BNG, Palmer AE, Tsien RY. Improved monomeric red, orange and yellow fluorescent proteins derived from Discosoma sp. red fluorescent protein. Nat Biotechnol. Nature Publishing Group. 2004;22:1567

48. Pelletier S, Gingras S, Green DR. Mouse Genome Engineering via CRISPRCas9 for Study of Immune Function. Immunity. 2015;42:18-27.

49. Muller WA, Teo R, Frank U. Totipotent migratory stem cells in a hydroid. Dev Biol. 2004;275:215-24.

50. Gild S, Frank U, Mokady O. Allogeneic interactions in Hydractinia: is the transitory chimera beneficial? Int J Dev Biol. 2003;47:433-8.

51. Camacho C, Coulouris G, Avagyan V, Ma N, Papadopoulos J, Bealer K, et al. BLAST+: architecture and applications. BMC Bioinformatics. 2009;10:421.

52. Sievers F, Wilm A, Dineen D, Gibson TJ, Karplus K, Li W, et al. Fast, scalable generation of high-quality protein multiple sequence alignments using Clustal Omega. Mol Syst Biol. 2011;7:539.

53. Kim JH, Lee S-R, Li L-H, Park H-J, Park J-H, Lee KY, et al. High cleavage efficiency of a 2A peptide derived from porcine teschovirus-1 in human cell lines, zebrafish and mice. PLoS One. 2011;6:e18556.

54. Gelvatol mounting medium. Cold Spring Harb Protoc. 2006;doi: https://doi. org/10.1101/pdb.rec10252.

55. Schindelin J, Arganda-Carreras I, Frise E, Kaynig V, Longair M, Pietzsch T, et al. Fiji: an open-source platform for biological-image analysis. Nat Methods. 2012;9:676-82.

\section{Ready to submit your research? Choose BMC and benefit from:}

- fast, convenient online submission

- thorough peer review by experienced researchers in your field

- rapid publication on acceptance

- support for research data, including large and complex data types

- gold Open Access which fosters wider collaboration and increased citations

- maximum visibility for your research: over $100 \mathrm{M}$ website views per year

At BMC, research is always in progress.

Learn more biomedcentral.com/submissions 